\title{
FUNDAMENTAL ROLE OF THE H2A.Z C-TERMINAL TAIL IN THE FORMATION OF
} CONSTITUTIVE HETEROCHROMATIN

László Imre ${ }^{1}$, Péter Nánási ${ }^{1}$, Rosevalentine Bosire ${ }^{1}$, Ágota Csóti ${ }^{1}$, Kata Nóra Enyedi ${ }^{4}$, Gábor Mezö $^{4,5}$, Masayuki Kusakabe ${ }^{6}$, Juan Ausio ${ }^{3}$, Masahiko Harata ${ }^{2 \#}$, Gábor Szabó ${ }^{1 \#}$

${ }^{1}$ Department of Biophysics and Cell Biology, Faculty of Medicine, University of Debrecen, Debrecen, H-4032, Hungary.

${ }^{2}$ Laboratory of Molecular Biology, Graduate School of Agricultural Science, Tohoku University, Sendai, Japan.

${ }^{3}$ Department of Biochemistry and Microbiology, University of Victoria, Victoria, British Columbia, Canada.

${ }^{4}$ Eötvös Loránd University, Faculty of Sciences, Institute of Chemistry,

${ }^{5}$ Eötvös Loránd Research Network, Supported Research Groups, Research Group of Peptide Chemistry

${ }^{6}$ Biosignal Research Center, Kobe University, Kobe 657-8501, Japan

\#Corresponding author: szabog@med.unideb.hu;

\begin{abstract}
Nucleosome stability, a crucial determinant of gene regulation, was measured in a robust in situ assay to assess the molecular determinants of the stability of H2A.Z-containig nucleosomes. Surprisingly, a large fraction of H2A.Z detected by three different antibodies was released from the nucleosomes by salt together with $\mathrm{H} 3$, and was associated with $\mathrm{H} 3 \mathrm{~K} 9 \mathrm{me} 3$ but not with H3K27me3 marked nucleosomes. This unusual behavior relied on the presence of the unstructured C-terminal chain of the histone variant, rather than on isoform specificity, posttranslational modifications or binding of the reader protein PWWPA2, as determined using cell lines expressing only particular forms of the variant. In the absence of this tail, or upon addition of an excess of the tail peptide to the nuclei of control cells, the canonical H2A-like stability features were readily restored and most of the H2A.Z-containing nucleosomes left the periphery and ended up in scattered foci in the nuclei. Concomitantly, the H3K9me3-marked constitutive heterochromatin was also dispersed, what was accompanied by increased overall nuclease sensitivity and significantly enhanced binding of intercalating dyes to the DNA. The DT40 cells expressing the tailless H2A.Z showed marked differences in their gene expression pattern and were distinguished by compromised DNA damage response. Thus, interactions involving a short H2A.Z peptide chain simultaneously determine the stability and accessibility features of chromatin involving the nucleosomes containing this histone variant and the localization of these large chromatin regions in the nucleus. Our data suggest that H2A.Z can function in both heterochromatic and in euchromatic scenarios depending on the molecular interactions involving its $\mathrm{C}$-terminal unstructured tail, shedding light on the enigmatic double-faced character of this histone variant.
\end{abstract}




\section{INTRODUCTION}

H2A.Z (1) is an all-important histone variant involved in the regulation of diverse and basic cell functions, involving activating as well as repressive effects that have proved difficult to reconcile in a unified model. These include: transcription (2-6), DNA replication (7), heterochromatin organization $(3,6,8,9)$, DNA repair $(10,11)$, cell differentiation and cell cycle $(12,13)$, epithelial mesenchymal transition in embryonic development and cancer (14). H2A.Z is upregulated in melanoma, breast, prostate, colorectal, liver, bladder and lung cancer $(3,15)$, it is an important player in intestinal epithelial cell homeostasis (13), Wnt, Notch, Nanog, Gli1 signaling (13,16-18), in central nervous system development and functioning (19-22). H2A.Z has about $60 \%$ identity to canonical H2A and comprises approximately $5 \%$ of the total $\mathrm{H} 2 \mathrm{~A}$ histones in vertebrates $(1,6,23)$.

H2A.Z is loaded onto the chromatin by the Tip60/p400 (24) and SRCAP (25) chromatin remodeling complexes, while ANP32E and INO80 are responsible for its eviction in human cells $(3,26-28)$. In addition to the INO80 type remodelers, ISWI and CHD family protein complexes have also been implicated in the replication independent dynamics of the variant $(29,30)$.

Since the nucleosomal structure is, in general, repressive for transcription, replication and repair, the strategy of eukaryotic cells to regulate these activities involves de-repression by destabilizing or mobilizing particular nucleosomes; therefore, the stability of nucleosomes is of utmost regulatory importance and it is expected to be modulated by posttranslational modifications (PTMs), as well as histone variant composition (31). In this context, destabilizing effects are expected in the case of activating functions, while nucleosome stabilization would suit repressive roles. In a puzzling manner, there are observations suggesting that the presence of H2A.Z in the nucleosomes can increase (32-35), and others indicating that it may rather decrease (36-39) nucleosome stability. Perhaps those that suggest their destabilizing role in euchromatin are more convincing. For example, as revealed by MNase-Seq, H2A.Z binding destabilizes local nucleosome structure in ES cells, leading to decreased nucleosome occupancy and increased chromatin accessibility particularly at enhancers (12). A similar observation has been made recently, showing that $\mathrm{H} 2 \mathrm{~A} . \mathrm{Z}$ deposition creates wide ATAC-Seq positive promoter regions in mouse fibroblasts genome-wide (40). In other studies, unusually labile H2A.Z containing nucleosomes, i.e. sensitive to low concentration of $\mathrm{NaCl}$, were detected at the TSS region of transcriptionally active promoters (38). These labile nucleosomes were composed of H2A.Z together with histone variant H3.3 bordering nucleosome free regions. Decreased unwrapping of the +1 nucleosomes was found recently by MNase-X-ChIP-seq upon depletion of H2A.Z (41), and earlier upon depletion of the remodeler esBAF (30). The stability features of H2A.Z containing nucleosomes localized to the transcriptionally repressed heterochromatin $(8,42)$ have not yet been characterized to our knowledge.

Two isoforms of H2A.Z are known to exist, encoded by separate genes, which differ by only 3 amino acids: H2A.Z1 and H2A.Z2 (43,44). The two isotypes have indispensable and selective functions during development $(45,46)$ and appear to have distinct roles in different forms of cancer $(6,47)$. Crystallographic analysis (48) revealed structural differences within the histone fold domain that are without any apparent influence on the stability of the core particles harboring the different isoforms, even though they exhibit differential in vivo mobility features, as pointed out in (6). On the other hand, alternative splicing gives rise to the hypervariant H2A.Z2.2 which is 
shorter than H2A.Z1 or Z2 by 14 amino acids and markedly destabilizes the nucleosomes relative to H2A.Z1 or H2A (49).

Thus, although there is no clear understanding of the relationships between stability features of H2A.Z-containing nucleosomes and their presence and roles in euchromatin vs. heterochromatin, the destabilizing role of a short $\mathrm{C}$-terminal region of the variant has been recognized. Anticipating a valuable insight into the stability features of $\mathrm{H} 2 \mathrm{~A} . \mathrm{Z}$-containing nucleosomes when performing such analyses in close to native conditions of the chromatin and in the context of nuclear architecture, we made use of an in situ assay of nucleosome stability, QINESIn (Quantitative Imaging of Nuclei after Elution with Salt/Intercalators) (50). This quantitative imaging cytometry based assay delivers histone type, post-translational modification (PTM) and cell cycle phase specific information on the intrinsic/DNA topology-dependent stability features of nucleosomes consisting of native endogenous or ectopically expressed histones, in situ, in populations of individual nuclei. A unique genetic complementation system (51) was used, involving H2A.Z1 and Z2 double knock out (DKO) DT40 cell lines expressing either transduced H2A.Z1 or Z2, a nonacetylatable Z1 mutant or a truncated H2A.Z1 missing its C-terminal 9 amino acids $(43,51,52)$, to learn what role the related factors may have in determining nucleosome stability in vivo. We show that the unstructured $\mathrm{C}$-terminal tail, via its interaction with the $\mathrm{H} 3 \mathrm{H} 4$ tetrasome, determines not only the stability of H2A.Z-containing nucleosomes irrespective of isoform composition, relevant PTMs and reader binding, but also their intranuclear localization. Moreover, the presence or absence of the C-terminal tail impacts overall chromatin architecture.

\section{RESULTS}

The H2A.Z-containing nucleosomes exhibit intranuclear heterogeneity reflected also in their stability features.

Using QINESIn (Suppl. Fig.1), we have shown that H2A.Z in the majority of nucleosomes is unusually stably associated with the tetrasomes (Fig. 1A), exhibiting elution profiles typical of H3 or H4, as compared to canonical H2A or H2A.X, in every phase of the cell-cycle (Suppl. Fig. 2A). Interestingly, the stability of the H2A.Z containing nucleosomes proved to depend on which antiH2A.Z antibody was applied to visualize the histone variant: an Abcam antibody (and two others; see Suppl. Fig. 2B,D) apparently detect the unusually stable H2A.Z containing nucleosomes, while a Thermo Fisher Scientific antibody, the only one among those tested, detect nucleosomes harboring $\mathrm{H} 2 \mathrm{~A} . \mathrm{Z}$ that behaved like $\mathrm{H} 2 \mathrm{~A}$ (designated ZAbA and ZAbB, respectively); i.e. ZAbA and the two other antibodies recognize stable nucleosomes in the sense that they do not shed the dimers at salt concentrations below that range where the tetrasomes are evicted, while ZAbB detects nucleosomes which bind H2A.Z as if it was canonical H2A (Fig. 1B). We note that in QINESIn antibody labeling is after the salt elution step, i.e. the elution behavior of a histone cannot be affected by antibody specificity, valence, or clonality. Interestingly, the fluorescent proteintagged forms of H2A.Z1 and Z2 both share the destabilized character observed through the spectacles of ZAbB (Suppl. Fig. 2E). The two H2A.Z containing chromatin regions are located strikingly differently: the unusually stable ones are at the periphery, whereas those with the stability features of canonical nucleosomes are much more scattered or more centrally located in the nuclei (Fig. 1C), in accordance with the manufacturers' datasheets. 
The H3-like behavior of H2A.Z in the ZAbA-detected nucleosomes suggested that H2A.Z may be physically linked to the $\mathrm{H} 3 \mathrm{H} 4$ tetrasomes here. Indeed, in immune-crosslinking experiments (see strategy in Fig. 1D), when H2A.Z was cross-linked by ZAbA prior to salt addition to prevent its subsequent elution (as described in ref. (50) ), H3 marked with K9me3 typical for constitutive heterochromatin was immobilized together with H2A.Z, unlike H3 marked with K27me3, a PTM enriched in facultative heterochromatin (Fig. 1E and F, respectively). These data suggest a direct molecular association of $\mathrm{H} 2 \mathrm{~A} . \mathrm{Z}$ with $\mathrm{H} 3$ in the $\mathrm{H} 3 \mathrm{~K} 9$ me3-containing nucleosomes in constitutive rather than in facultative heterochromatin.

Thus, H2A.Z could be detected in nucleosomes residing in the peripheral heterochromatin, exhibiting unusual salt resistance, i.e. stability, and also in nucleosomes of canonical salt elution profiles and a more homogenous topography. Heterogeneity of the H2A.Z-containing nucleosomes was also observed using ethidium bromide (EBr, a DNA intercalator) as a destabilizing agent (50). In this case the H2A.Z elution profile (detected by ZAbA) was similar to that of the canonical H2B, however, a major intercalator-resistant fraction remained (Fig. 2A) independently of cell cycle phases (Suppl. Fig. 2F, G). This residual exhibited a heterochromatinlike peripheral and perinucleolar H2A.Z staining pattern (Fig. 2B), suggesting that H2A.Z exhibiting relative salt-resistance in terms of binding to the tetrasome remained confined to the original location of constitutive heterochromatin. This impression was confirmed when the EBr resistant nucleosome fraction was further analyzed by mass spectrometry (MS) (following the experimental scheme shown in Fig. 2C), detecting proteins typical of constitutive heterochromatin, similarly to the sample where the first elution step involved elution with $1.2 \mathrm{M}$ salt instead of $\mathrm{EBr}$ together with $750 \mathrm{mM}$ salt (50) (Fig. 2D). At $1.2 \mathrm{M}$ salt concentration most of the relatively unstable H2A.Z-containing nucleosomes (detectable by ZAbB) disassemble, as Fig. 1A and B shows. Thus, both pretreatments appear to release H2A.Z from the nucleosomes exhibiting the canonical, H2A-like stability features, leaving the more stable ones heterochromatin-associated. In further experiments, H2A.Z isotype composition, PTMs (acetylation, ubiquitination and sumoylation) that potentially mark H2A.Z, and the presence or absence of the C-terminus binding reader protein PWWP2A (52) were tested to determine if they affect the stability of nucleosomes containing H2A.Z, as manifested in the salt or intercalator elution profiles. These studies, employing specific inhibitors and cell lines expressing different isoforms or mutants of $\mathrm{H} 2 \mathrm{~A} . \mathrm{Z}$ in a double KO (DKO) DT40 background (51) and cells expressing PWWP2A at different levels, have shown that the stability features measured do not depend on H2A.Z isotype specificity, acetylation, ubiquitination or sumoylation (Suppl. Fig. 2B-D, Suppl. Fig. 3); the finding that the H2A.Z elution curves were similar at different PWWP2A expression levels is not surprising in view of the fact that the reader is eluted at rather low salt, so it could not affect the stability features measured in our assay (Suppl. Fig. 3F-H).

\section{Effect of the H2A.Z C-terminus on nucleosome stability and nuclear architecture.}

In view of the fact that the C-terminal region of the unstable H2A.Z.2.2 isoform is 14 residues shorter than H2A.Z (31), where the last six residues of Z.2.2 form a motif facilitating nucleosome assembly (53), we conducted experiments with cells expressing H2A.Z carrying just a 9 amino acid deletion at the end of their C-terminus (between amino acids 119-128), on a DKO DT40 background (51). The H2A.Z $\Delta \mathrm{C}$ containing nucleosomes were destabilized as compared to fulllength histones (Fig. 2E, Suppl. Fig. 4A), while the stability of tetrasomes carrying H3K9me3 or 
H3K27me3 were not affected significantly (Suppl. Fig. 4B, C). The destabilized character of $\mathrm{H} 2 \mathrm{~A} . \mathrm{Z} \Delta \mathrm{C}$ nucleosomes was also apparent in intercalator elution experiments, as the plateau corresponding to the EBr resistant nucleosomes decreased in these cells (Suppl. Fig. 4D).

Not only the stability of H2A.Z containing nucleosomes but also their nuclear localization pattern has proved to be C-terminus dependent (Fig. $2 \mathrm{~F}$ ). In the H2A.Z $\Delta \mathrm{C}$ expressor cells, the typically heterochromatic localization of H2A.Z is exchanged for a much more uniform, more central topography in the same DKO background, using the same ZAbA antibody. Furthermore, the peripheral localization of $\mathrm{H} 3 \mathrm{~K} 9 \mathrm{me} 3$ marked nucleosomes has also changed, as reflected by the altered texture of $\mathrm{H} 3 \mathrm{~K} 9 \mathrm{me} 3$ containing structural elements (Fig. 2G) and by the changed colocalization between H3K9me3 and H3K27me3 (Suppl. Fig. 5B). The degree of olocalization between $\mathrm{H} 2 \mathrm{~A} . \mathrm{Z}$ and $\mathrm{H} 3 \mathrm{~K} 9 \mathrm{me} 3$ was significantly lower in the H2A.Z $\Delta \mathrm{C}$ than in the case of H2A.Z1 DKO nuclei (Fig. 2H), while the difference was much smaller in the case of H2A.Z and H3K27me3 (Suppl. Fig. 6B). The conspicuous decrease of colocalization between H2A.Z and $\mathrm{H} 3 \mathrm{~K} 9 \mathrm{me} 3$ is present in spite of the fact that H2A.Z $\Delta \mathrm{C}$ levels are smaller than those of H2A.Z (Suppl. Fig. 4E). As an opposite example, the higher Manders' coefficient indicative of more H2B colocalizing with H2A.Z in the $\Delta \mathrm{C}$ nuclei relative to those harboring the full-length form (Suppl. Fig. 5C) can be explained by the decrease in H2A.Z levels, but not of H2B, in these nuclei. At the same time there was no change in the colocalization coefficients in the case of PWWP2A and H2B (Suppl. Fig. 5D). H2A.Z localization was also different when compared to that of the H1 linker histone or HP1 (Suppl. Fig. 6E, F). In contrast with the above observations on ZAbA-detected H2A.Z (likely hererochromatic based on the data described herein), when the variant was labeled using ZAbB (likely euchromatic based on our data), no significant differences were measured between the two kinds of nuclei in terms of the colocalization of the histone variant with either the euchromatic mark H3K4me3 or the heterochromatic mark H3K27me3 (Suppl. Fig. 6B, D). On the other hand, the fraction of ZAbB-detected H2A.Z colocalizing with H3K9me3 decreased significantly in the $\Delta \mathrm{C}$ nuclei (Suppl. Fig. 6A), suggesting that this antibody also recognizes nucleosomes residing in the peripheral heterochromatin. In line with this notion, ZAbB also stains nucleosomes at the nuclear periphery, albeit exhibiting a different topography (Suppl. Fig. 6A, B). Summarily, the nucleosomes containing the ZAbA-detected H2A.Z, but not those with the ZAbBdetected ones, are differently arranged within the nuclei of DT40 cells harboring full-length H2A.Z1 or its truncated version, moreover the two kinds of nuclei also exhibit differences in the topography of nucleosomes not harboring the variant.

These architectural differences are reflected in global effects of functional consequences, as shown below. As compared to DKO H2A.Z nuclei, those of the DKO H2A.Z $\Delta \mathrm{C}$ line stained much more readily with intercalator dyes used at such low concentrations where dye binding is DNA topology dependent (54), and were much more readily digested using MNase, nickase and DNAse I (Fig. 3), suggesting major H2A.Z C-terminal tail-dependent overall differences in chromatin structure. The $\mathrm{H} 2 \mathrm{~A} . \mathrm{Z} \Delta \mathrm{C}$-containing nucleosomes were also less stable than those containing $\mathrm{H} 2 \mathrm{~A} . \mathrm{Z}$ using doxorubicin instead of $\mathrm{EBr}$; this clinically relevant intercalator was able to evict $\mathrm{H} 2 \mathrm{~A} . \mathrm{Z} \Delta \mathrm{C}$ from the nucleosomes while not affecting the tetrasomes harboring H3K27me3 in the same sample of nuclei (Suppl. Fig. 7A, B).

These changes in nuclear architecture and accessibility features are accompanied by dramatic changes of gene expression patterns, when wild-type (WT) and DKO H2A.Z cells were compared with DKO H2A.Z $\Delta$ C (Suppl. Fig. 7C-F). Among the genes substantially up- or down-regulated in 
DKO H2A.Z $\Delta$ C relative to DKO H2A.Z or WT, those that have been implicated with pathways where H2A.Z is known to participate are listed in Suppl. Fig. 8. These include processes directly involving H2A.Z, like DNA repair $(10,11)$. Indeed, DKO H2A.Z $\Delta$ C cells appear to exhibit a diminished DNA damage response and relatively poor survival upon exposure to DNA damaging agents (Suppl. Fig. 9).

Remarkably, the destabilized character and altered intranuclear distribution of nucleosomes containing H2A.Z $\Delta \mathrm{C}$ were both reproduced when a peptide representing the last 9 amino acids of the C-terminus was added to the nuclei (Fig. 4A-D, Suppl. Fig. 10A-C), suggesting that these differences are the direct consequence of molecular interactions involving the tail region. The $\mathrm{H} 3 \mathrm{~K} 9 \mathrm{me} 3$-marked constitutive heterochromatin was also rearranged, as its texture distribution has changed in several size regions of pixels (Suppl. Fig. 10C). At the same time, the H2A.Zcontaining nucleosomes became partially separated from the H3K9me3-rich periphery based on the significantly decreased Manders' coefficient (Suppl. Fig. 10B).

\section{DISCUSSION}

The data demonstrating the decisive role of the C-terminal unstructured tail of H2A.Z both in the stability features of nucleosomes harboring this variant and in their localization call for interpretation in several contexts, including (a) nucleosomal structure, (b) nuclear organization of constitutive heterochromatin and (c) the accessibility of chromatin.

We have found that the H2A.Z-containing nucleosomes detected by ZAbA are unusually stable in the sense that H2A.Z is evicted from the nucleosomes at much higher salt concentrations than typical for H2A. As Suppl. Fig. 10D, E demonstrate, the H3-like stability readily reverted to H2Alike in both the salt and the intercalator elution format of the assay when the nuclei were pretreated with nickase; topological relaxation via nicking was previously shown by us to destabilize nucleosomes (50). This result together with the fact that the H2A.Z-containing nucleosomes behaved in the same way through the spectacles of two other antibodies (Suppl. Fig. 2B,D) show that the stability related phenomenon is not an antibody related artifact. Furthermore, the unusually stable nucleosomes become destabilized to resume their canonical state when the C-terminal tail is deleted or its binding to molecular partners is prevented by the addition of a competing peptide. These observations are in agreement with earlier findings showing that the unstructured $\mathrm{C}$-terminal end of $\mathrm{H} 2 \mathrm{~A} . \mathrm{Z}$ is responsible for the $\mathrm{H} 2 \mathrm{~A}-\mathrm{H} 2 \mathrm{~B}$ dimer - $\mathrm{H} 3 \mathrm{H} 4$ tetramer interaction within the nucleosome and is crucial for the normal function of H2A.Z in both yeast and mammalian cells $(55,56)$. The fluorescent protein tags apparently prevent this interaction (Suppl. Fig. 2E). In contrast with ZAbA, ZAbB detects nucleosomes with stability features similar to those of the canonical nucleosomes. Based on the localization patterns (see e.g. Fig. 1C), the tail-dependent changes and differences thereof (e.g. as shown in Fig. 2C-H, Fig. 4B-D) and the stability features (Fig. 1B, Fig. 4A) we propose that ZAbA detects nucleosomes residing in constitutive heterochromatin, while $\mathrm{ZAbB}$ recognizes the histone variant mainly in nucleosomes belonging to euchromatin.

This scenario is also in line with the lack of structural features suggestive of significant destabilizing interactions in the H2A.Z1-containing nucleosomes relative to those carrying $\mathrm{H} 2 \mathrm{~A}$ (48). A large, ladle-like $\mathrm{C}$-terminal region of $\mathrm{H} 2 \mathrm{~A}$ interacts with the $\mathrm{N}$-terminus of $\mathrm{H} 3$ what is only slightly affected by a Gln-Gly substitution resulting in the loss of three hydrogen bonds in the 
case of H2A.Z; based on this and the other structural features of H2A.Z-containing nucleosomes, no severe destabilization was anticipated (48). The possibility that the difference in manganese binding between H2A and H2A.Z may perhaps lead to differences in stability was not assessed in our study employing EDTA to assure DNA integrity, but this factor could not account for the taildependent differences anyway. In the same paper, pairing of one H2A.Z and one H2A chain within the same nucleosome core particle was predicted to lead to steric clashes, so we consider uniformly homotypic (H2A.Z-H2A.Z) dimers in the nucleosomes (as in our model shown in Fig. 5; see below).

Based on recent cryo-EM analyses (53), a H2A motif coined "regulating-octamer-folding" (ROF) plays an important role in nucleosome stability, interacting with the N-terminal $\mathrm{H} 3$ helix. This motif, residing between 106-111, is present both in H2A and H2A.Z1. In the alternatively spliced variant $\mathrm{H} 2 \mathrm{~A}$ Z2.2 it is replaced by a noncanonical ROF and the terminal 14 amino acids are also missing as compared with H2A.Z1. The destabilized character of H2A.Z2.2-containing nucleosomes (49) was attributed primarily to the disruptive effect of the noncanonical ROF in (53). The contribution to stability of the last 14 amino acids missing from $\mathrm{Z} 1$ was not possible to judge since this segment (encompassing the noncanonical ROF) is invisible in cryo-EM. The H2A.Z1 $\Delta \mathrm{C}$ cells expressing a truncated H2A.Z1 that is shorter by 9 C-terminal amino acids but still contains a canonical ROF seems suitable to investigate the contribution of the unstructured tail to the stability of nucleosomes. In the lack of the C-terminal tail, H2A.Z2.2 cannot be linked to H3 in the H3K9me3-marked nucleosomes, hence the disruptive effect of the noncanonical ROF is expected to become manifest. The C-terminal tail beyond amino acids 118-122 was not resolved in neither the cryo-EM (53) nor the crystallographic study (48) due to its unstructured character, but its binding to $\mathrm{H} 3$ might not have been observed anyway in the absence of the K9me3 modification (see Fig. 1E,F). The C-terminal 9 amino acids of H2A.Z apparently mediate an unusually stable association of $\mathrm{H} 2 \mathrm{~A}$. $\mathrm{Z}$ with the $\mathrm{H} 3 \mathrm{~K} 9 \mathrm{me} 3$ nucleosomes. The destabilized character of the H2A.Z2.2-containing nucleosomes was not tested in our work.

Reader proteins such as PWWP2A (3), and chaperones/remodelers $(3,11,26,57,58)$ can bind to the $\mathrm{H} 2 \mathrm{~A} . \mathrm{Z} \mathrm{C}$-terminus preventing it from engaging $\mathrm{H} 3$, raising the possibility that binding of these factors to H2A.Z can modulate the stability of H2A.Z containing nucleosomes. In addition, posttranslational modifications targeting the same region (59) could also play a role. However, these factors appear not to play a role in our observations (Suppl. Fig. 2D, Suppl. Fig. 3C-H). PWWP2A is evicted at such low salt concentrations where H2A.Z still remains associated with the nucleosomes (Suppl. Fig 3F). Furthermore, in PWWP2A-GFP expressing cells, the stability of H2A.Z nucleosomes, detected by ZAbA, was independent from the level of (exogeneous) PWWP2A expression (Suppl. Fig. 3G, H), thus binding of this reader protein cannot be an important determinant of the nucleosome stability features assessed in our assay system. The possibility that in live cell conditions PWWP2A (or other readers/chaperones/remodelers not tested in our work) may disengage the tails from their $\mathrm{H} 3$ binding could not be assessed in our system relying on elution measurements in nuclei, and seems a very plausible scenario.

The indistinguishable elution profiles of fluorescent protein-tagged H2A.Z1 and Z2 (Suppl. Fig. 2B, C, E, Suppl. Fig. 3A, B) suggests that the two hypervariants may not differ significantly in their stability features. In view of their $\mathrm{H} 2 \mathrm{~A}$-like conduct, the tag apparently prevents incorporation of the fusion proteins into constitutive heterochromatin. This is not surprising in view of the fact that even a Flag tag can have profound effects on the molecular interactions involving e.g. H2B (60). 
We did not see any significant variation in the affinity of $\mathrm{H} 2 \mathrm{~A} . \mathrm{Z}$ binding to nucleosomes when the global extent of histone acetylation was increased by treatment with the HDAC inhibitors (Suppl. Fig. 3C) or a non-acetylatable H2A.Z mutant was expressed in the cells (Suppl. Fig. 10F), or using an antibody specific for acetylated H2A.Z (Suppl. Fig. 2D). Thus, we attribute the increased stability of H2A.Z-containing ZAbA-detected nucleosomes to the C-terminal tail rather than any of these factors. The fact that the C9 peptide efficiently modulates stability of these nucleosomes suggests that the molecular interactions involving the tail rather than the tail itself, determine stability.

The experiments involving addition of the $C 9$ peptide to nuclei also reveal that the differences between the topography of H2A.Z and H3K9me3 in DKO H2A.Z and H2A.Z $\Delta \mathrm{C}$ nuclei are the direct result of the altered structure and functioning of the histone variant, rather than secondary, e.g. compensatory, consequences thereof. Our data lend a strong support to the assumption that H2A.Z is involved in the organization of constitutive heterochromatin (8), and point out the role of the $\mathrm{C}$-terminal tail in the molecular interactions involved. In view of the fact that nucleosome destabilization, from stable to canonical (Fig. 2E), is accompanied by the detachment of H2A.Zcontaining chromatin from the lamina (Fig. 2F), we hypothesize that the proper presentation of H3K9me3 for HP1 chromodomain binding (61-63) is secured by the reclining C-terminal tail of H2A.Z, if present (DKO H2A.Z cells) and if the tail is not competed out by the exogenous C9 peptide added in excess, as shown in the model of Fig. 5. The basic tenet of the model, i.e. the role of the unstructured C-terminal tail in binding of H2A.Z to the rest of the nucleosome, is in line with the lower stability of the C-terminally truncated splice variants in both yeast and human cells $(49,55)$.

We note that the model shown in Fig. 5 is not conform with the observation that H3 and H2A.Z were not co-eluted by $\mathrm{EBr}$, as opposed to their simultaneous eviction by salt. This finding can be interpreted to suggest that the C-terminal H2A.Z tail may actually bind the linker DNA and that this binding is overcome by the competing intercalating agent. It is also possible that the tailmediated component of the H2A.Z-H3 interactions is superhelicity dependent. These alternative scenarios have not been further analyzed in this work. The EBr resistance of a fraction of H2A.Z staying nucleus confined in spite of the $\mathrm{H} 3 \mathrm{H} 4$ tetrasomes being quantitatively eluted already may suggest that a fraction of $\mathrm{H} 2 \mathrm{~A} . \mathrm{Z}$ stays engaged in macromolecular interactions even after the $\mathrm{H} 3 \mathrm{H} 4$ tetrasomes are evicted. Constitutive heterochromatin may qualify as such a network. In this scenario H2A.Z collaborates with HP1 in an H1-dependent manner to maintain the H3K9me3 marked constitutive heterochromatin in a lamina-tethered, phase-separated state $(8,64-66)$. According to ref. (8), HP1 $\alpha$ interacts with the linker DNA unoccluded by linker histones and also with the nucleosome core, and this latter mode of binding is augmented by both $\mathrm{H} 2 \mathrm{~A} . \mathrm{Z}$ and $\mathrm{H} 3 \mathrm{~K} 9 \mathrm{me} 3$. Indeed, pairwise interactions involving these molecular players have been independently demonstrated: the N-terminal tail of $\mathrm{H} 3$ influences the conformation of the unstructured CTD of H1 by direct interaction of the two (67); HP1 and H2A.Z may also directly interact (68). The strikingly ready reorganization of the constitutive heterochromatin upon addition of C9 fits well the concept of phase-separated domains involving the interaction of unstructured peptide chains that are easily peeled away one-by-one $(65,69)$._A more complex model including $\mathrm{H} 1$ in the picture, based on the observation that the C-terminal tail of H2A.Z disfavors $\mathrm{H} 1$ binding $(70,71)$, is not considered in view of the fact that $\mathrm{H} 1$ appears to accompany H2A.Z in the DKO H2A.Z as well as in DKO H2A.Z $\Delta$ C cells (Suppl. Fig 6E); furthermore, that observation was also challenged based on data obtained with alternative methods (72). 
Together with H3K9me3 and HP1, H2A.Z takes part in the formation of the centric and pericentric heterochromatin (73); whether the $\Delta \mathrm{C}$ state or addition of the $\mathrm{C} 9$ peptide affects the centromere structure has not been explored in this work.

H2A.Z is also present in facultative heterochromatin marked with H3K27me3 (74). The lack of molecular proximity of the two entities demonstrated in our cross-linking experiments (Fig. 1F) suggests that they are not in the same nucleosome in that heterochromatic environment.

Making use of the fact that the unusually stable association of H2A.Z with a major fraction of the nucleosomes could be revealed also through the spectacles of intercalator elution, we could analyze the intercalator resistant fraction by mass spectrometry (Fig. 2B, C), confirming the association of these nucleosomes with constitutive heterochromatin. The fact that ZAbA itself binds with a different (higher) affinity to the EBr-resistant subpopulation, as compared to the binding affinity measured for all the epitopes present before exposure to the intercalator (Suppl. Fig. 10G), is in line with the different conformation of H2A.Z in its two roles.

The conformational heterogeneity of $\mathrm{H} 2 \mathrm{~A} . \mathrm{Z}$ as revealed through the spectacles of the antibodies was apparent both in terms of the intrinsic stability of nucleosomes assessed via salt elution, and also in terms of their intercalator resistance; however, the two features do not usually coincide (50) and the exact relationship of the two kinds of heterogeneities was not further explored. The list of proteins that remain associated with chromatin after treatment with $1.2 \mathrm{M}$ salt or with 100 $\mu \mathrm{g} / \mathrm{ml} \mathrm{EBr}+750 \mathrm{mM}$ salt partially overlap, with exceptions including $\mathrm{H} 1$ species resisting the intercalator but readily eluted by salt (Fig. 2D). H2A.Z, although clearly visible after $\mathrm{EBr}$ at the nuclear periphery and also perinucleolarly (Fig. 2B), was not detected by MS in the high salt eluted sample of the EBr treated nuclei (Fig. 2D), what is attributed to the presence of residual $\mathrm{EBr}$ that could not be removed completely from the samples and therefore affected MS analyzes; in general, fewer proteins were detected in the $\mathrm{EBr}$ treated.

The dramatic reorganization of peripheral heterochromatin upon addition of C9 may not necessarily imply that the H2A.Z-containing nucleosomes are tethered to the periphery directly involving the variant's C-terminal tail. In an alternative scenario, H2A.Z may affect HP1's switch to a crosslinking-competent conformation (75), what could influence the formation and tethering of the phase-separated heterochromatin. However, the observation that the localization of HP1 appears to be invariant to the truncation of the tail (Suppl. Fig. 6F) does not support this hypothesis.

The remarkable differences in overall nuclease sensitivity between the DKO H2A.Z and H2A.Z $\Delta \mathrm{C}$ nuclei (Fig. 3) may reflect the altered contribution to what is measured over the entire nucleus of a disassembled constitutive heterochromatin, and/or accessibility changes encompassing other chromatin regions. In the latter scenario H2A.Z $\Delta$ C, present only at its promoter proximal locations, would render these sites more accessible to nucleases. Such a scenario is in good agreement with the report that chromatin accessibility is increased genome-wide at promoter regions by $\mathrm{H} 2 \mathrm{~A} . \mathrm{Z}$ (40), what is antagonized by ANP32E, the chaperone catalyzing its eviction engaging the Cterminal tail of H2A.Z (26). It will be of interest to determine which scenario explains the striking global differences.

The large difference between the staining of the DKO H2A.Z and H2A.Z $\Delta$ C nuclei with the two intercalating dyes would be unexpected if the dyes were applied at the usual concentration allowing 
for stoichiometric binding to the DNA. However, they were added at a lower concentration where their binding to the DNA is highly sensitive to the constrtained nucleosomal and to the plectonemic internucleosomal superhelicity (54). The H2A.Z $\Delta \mathrm{C}$ levels expressed in the nuclei of these DKO DT40 cells are about half of the H2A.Z levels in the same DKO background, but the total number of $\mathrm{H} 3 \mathrm{~K} 9 \mathrm{me} 3, \mathrm{H} 3 \mathrm{~K} 27 \mathrm{me} 3, \mathrm{H} 2 \mathrm{~B}$ nucleosomes and also the amount of DNA in the nuclei of the two cell types are similar (Suppl. Fig. 4E). On the other hand, the ZAbB-detected H2A.Z-containing nucleosomes may reside in topologically relaxed chromatin (Suppl. Fig. 10D, E), what makes us speculate that increased intercalator uptake may be a consequence of $\mathrm{H} 2 \mathrm{~A} . \mathrm{Z}$ being incorporated into euchromatin rather than constitutive heterochromatin (76).

The architectural changes involving DKO H2A.Z $\Delta \mathrm{C}$ chromatin as well as sensitivity of these cells to etoposide and doxorubicin (Suppl. Fig. 9) are closely reminiscent of the dramatic consequences of H2A.Z(1-114) truncation in Saccharomyces cerevisiae on resistance to genotoxic stress and restriction of the spread of heterochromatin at boundaries with euchromatin, and nucleosomal binding (56). The special significance of the C-terminus in these functions was also supported independently by mutational analyses (77).

The differences in gene expression between the DKO H2A.Z and H2A.Z $\Delta \mathrm{C}$ cells are extensive (Suppl. Fig. 7C-F Suppl. Fig. 8), in line with the global contribution of H2A.Z to gene expression (51), and are in line with the massive differences in chromatin structure both in terms of nuclear localization and accessibility features in the cycling cells of the same cell line (Fig. 2E-H, Fig. 3). Whether H2A.Z plays an important role in the transcriptional regulation of resting, differentiated cells as well, is uncertain; the variant appears to regulate the activity-induced neuronal gene transcription in differentiating cultures of cortical neurons (20) but seems dispensable for both basal and activated transcription in postmitotic muscle cells (78). Based on the above, the cell cycle block elicited by the DNA damaging agents used in our experiments may not have contributed to the increased cell death of $\mathrm{H} 2 \mathrm{~A} . \mathrm{Z} \Delta \mathrm{C}$ cells.

Heterochromatin comprises about $40 \%$ of the human genome and its loss or alterations appear to be relevant for aging, to various neurological disorders, and are thought to predispose for carcinogenesis (79). The apparent dispersal of peripheral heterochromatin upon treatment of nuclei with the $\mathrm{C} 9$ peptide may demonstrate that these global features of chromatin structure are amenable to modulation by peptides what could also be targeted to particular cell types. Changes elicited in nuclear architecture would probably lead to dramatic changes also in gene expression patterns based on the differences detected by microarray analyses between the DKO H2A.Z and the H2A.Z $\Delta$ C cells (Suppl. Fig. 7C-F Suppl. Fig. 8).

The chromatin remodeling steps following double-strand break induction involve sequential H2A.Z deposition and removal (80); in view of the waned DDR of the $\Delta \mathrm{C}$ cells in response to DNA damaging agents (Suppl. Fig. 9), its C-terminus plays an important role in this function of the histone variant. Proteins belonging to the Fanconi anemia pathway, what is involved in DDR elicited by topoisomerase II poisons (81), were expressed in a H2A.Z C-terminal tail-dependent manner (Suppl. Fig. 8), potentially explaining the relative vulnerability of H2A.Z $\Delta \mathrm{C}$ cells to such drugs (Suppl. Fig. 9). The role of H2A.Z in DNA repair may also be related to its functioning in the retention of transiently stalled replication forks, based on yeast analogy (82). The findings presented raise the possibility of modulating DDR using C9-like peptides reaching their specific target sites in the nuclei of live cells. We note that the H2A.Z $\Delta \mathrm{C}$ levels being lower than those of H2A.Z (Suppl. Fig. 4E), such a quantitative difference may also account for the differences in 
DDR (Suppl. Fig 9). However, the $\gamma \mathrm{H} 2 \mathrm{~A} . \mathrm{X}$ response was very similar in the nuclei gated for different expression levels of H2A.Z. (Suppl. Fig. 9B-D).

In summary, the enigmatic involvement of H2A.Z in both gene activating and silencing processes appears to be mainly regulated via the molecular interactions of its unstructured C-terminal tail. This tail links the variant histone to $\mathrm{H} 3$ in the H3K9me3-marked nucleosomes of constitutive heterochromatin, increasing nucleosome stability and also shielding the variant from engagement with chaperones/remodelers targeting the tail. Both the H2A.Z tail-H3 linkage and the peripheral location of heterochromatin can be reversed by the addition of the purified terminal peptide to nuclei, The profound differences between the chromatin of DT40 cells harboring full length or the $\triangle \mathrm{C} \mathrm{H} 2 \mathrm{~A} . \mathrm{Z}$ variant in terms of DNA accessibility features assessed at the level of the individual nuclei, concomitant with changes in the gene expression pattern and with functional consequences in DDR, raise the possibility that these cellular features could be modulated introducing appropriate derivatives of the $\mathrm{C}$-terminal peptide into live cells.

\section{MATERIALS AND METHODS}

\section{Chemicals}

All reagents were from Sigma-Aldrich (St. Louis, Missouri, USA) unless otherwise stated.

\section{Plasmids}

The plasmids carrying different isoforms of the histone variant H2A.Z (provided by Juan Ausio) were transformed into Escherichia coli DH5 $\alpha$ by heat shock and selected on LB plates containing $100 \mu \mathrm{g} / \mathrm{ml}$ kanamycin.

\section{Cells}

HeLa cells expressing H2B-GFP and H3-GFP fusion proteins (83), and HCT116 cells (Developmental Therapeutics Branch, National Cancer Institute, Bethesda, Bethesda, MD, 20892) were cultured in DMEM supplemented with 10\% FCS, $2 \mathrm{mM}$ l-glutamine, $100 \mu \mathrm{g} / \mathrm{ml}$ streptomycin, $100 \mathrm{U} / \mathrm{ml}$ penicillin. Wild type, H2A.Z1 knock-out, H2A.Z2 knock-out and mutant (5KR and $\Delta \mathrm{C}$ ) H2A.Z1 expressing double knock-out DT-40 chicken B cells (provided by Masahiko Harata $(51,52)$ ) were cultured in DMEM supplemented with $2 \%$ chicken serum, $8 \%$ FCS, $2 \mathrm{mM}$ l-glutamine, $100 \mu \mathrm{g} / \mathrm{ml}$ streptomycin, $100 \mathrm{U} / \mathrm{ml}$ penicillin.

\section{Embedding live cells into low melting point agarose}

Prior to embedding, the wells of 8-well chambers (Ibidi, Martinsried, Germany) were coated with $1 \%(\mathrm{~m} / \mathrm{v})$ low melting point (LMP) agarose. $150 \mu \mathrm{l}$ liquid agarose diluted in distilled water was dispensed into each well and was immediately removed so that a thin agarose layer remained on the surfaces and was left to polymerize on ice for 2 minutes, then kept at $37^{\circ} \mathrm{C}$ until the surface of the wells dried out. This coating procedure was repeated once more on the same chambers. Embedding was performed keeping cells and agarose at $37^{\circ} \mathrm{C}$. The cell suspension containing $6 \times 10^{6}$ cells $/ \mathrm{ml}$ was mixed with $1 \%$ LMP agarose diluted in $1 \times \mathrm{PBS}$ at a $\mathrm{v} / \mathrm{v}$ ratio of $1: 3.22 \mu 1$ of 
the cell-agarose suspension was dispensed in the middle of the wells and the chambers were covered with home-made rectangular plastic coverslips cut out from a $200 \mu \mathrm{m}$ thick, medium weight polyvinyl chloride binding cover (Fellowes, Inc., Itasca, Illinois, USA). Cells were left to sediment on the surface of the coated wells for 4 minutes at $37^{\circ} \mathrm{C}$, then kept on ice for 2 minutes. After polymerization of the agarose, $300 \mu$ ice cold complete culture medium was added to each well, a step aiding removal of the coverslips.

\section{Preparation of nuclei/permeabilization and histone eviction by salt or intercalators}

The agarose embedded cells at the bottom of the wells were washed with $500 \mu$ ice cold $1 \times \mathrm{PBS}$, 3 times for 3 minutes each, then permeabilized with $500 \mu$ ice cold 1\% (v/v) Triton X-100 dissolved in $1 \times$ PBS/EDTA ( $5 \mathrm{mM}$ EDTA in PBS), for 10 minutes. This step was repeated once more. After permeabilization, nuclei were washed with $500 \mu \mathrm{l}$ ice cold $1 \times$ PBS/EDTA 3 times for 3 minutes and were treated with different concentrations of $\mathrm{NaCl}$ or intercalator solutions on ice. Ethidium bromide (EBr) was diluted in $1 \times \mathrm{PBS} / \mathrm{EDTA}$ supplemented with $600 \mathrm{mM} \mathrm{NaCl}$, to the final concentrations indicated in the Figures. Doxorubicin (TEVA, Debrecen, Hungary) was diluted in $1 \times$ PBS/EDTA. Nuclei were incubated with $500 \mu \mathrm{l}$ of ice cold salt or intercalator solution for 60 minutes. After this treatment, nuclei were washed with $500 \mu$ ice cold $1 \times$ PBS/EDTA 3 times for 3 minutes. Since $\mathrm{NaCl}$ was diluted in $1 \times \mathrm{PBS} / \mathrm{EDTA}$, the salt concentrations indicated on the $\mathrm{X}$ axes of the graphs in all the Figures show the total $\mathrm{NaCl}$ concentrations together with $\mathrm{NaCl}$ present in the PBS buffer. For the analyzes of the curves SigmaPlot 12.0 software was applied, using either the 'Sigmoid 3 parameter' (in the case of linear plots) or 'Standard curves: Four Parameter Logistic Curve' (in the case of logarithmic plots) curve-fitting subroutines. Elution curves were normalized to ' 0 ' substracting the smallest value from all the others, and to ' 1 ' dividing the mean fluorescence intensities represented by the data points by that of the non-treated sample. The number of analyzed G1 nuclei were between 200-2000/well, out of the about 500-5000 cells scanned. All the SEM values indicated in the Figures were calculated from the datapoints of the cell population analyzed in the given experiment.

\section{Immunofluorescence labeling}

After salt or intercalator treatment the samples were incubated with $500 \mu 15 \%$ (m/v) Blotto NonFat Dry Milk (Santa Cruz Biotechnology Inc., Santa Cruz, California, USA) in $1 \times$ PBS/EDTA for 30 minutes on ice, to decrease nonspecific binding of the antibodies. The blocking solution was washed out with $500 \mu \mathrm{l}$ ice cold $1 \times$ PBS/EDTA 3 times for 3 minutes and indirect immunofluorescence labeling was performed using rabbit polyclonal anti-H2A.Z (ab97966 (ZAbA), ab4174, Abcam, Cambridge, UK; $1 \mathrm{mg} / \mathrm{ml}$ ), sheep polyclonal anti-H2A.Z (acetyl K4+K7+K11, ab18262, Abcam, Cambridge, UK; 0.5 mg/ml), rabbit polyclonal anti-H2A.Z (PA517336 (ZAbB), Thermo Fisher Scientific, Waltham, Massachusetts, USA), rabbit polyclonal antiPWWP2A (NBP2-13833, Novus Biologicals, Centennial, Colorado, USA; $0.2 \mathrm{mg} / \mathrm{ml}$ ), rabbit polyclonal anti-H2A.X (ab11175, Abcam, Cambridge, UK; $1 \mathrm{mg} / \mathrm{ml}$ ), rabbit polyclonal anti-H2A (ab18255, Abcam, Cambridge, UK; $1 \mathrm{mg} / \mathrm{ml}$ ), rabbit polyclonal anti-H2B (ab52484, Abcam, 
Cambridge, UK; $1 \mathrm{mg} / \mathrm{ml}$ ), mouse monoclonal anti-H1 (ab71591, Abcam, Cambridge, UK; 1 $\mathrm{mg} / \mathrm{ml}$ ), mouse monoclonal anti-HP1 (ab234085, Abcam, Cambridge, UK; $1 \mathrm{mg} / \mathrm{ml}$ ), mouse monoclonal anti- $\gamma$ H2A.X (05-636, Merck-Millipore, Darmstadt, Germany), mouse monoclonal anti-H3K4me3 ((84); 0,5 mg/ml), mouse monoclonal anti-H3K9me3 ((84); 0,5 mg/ml) or mouse monoclonal anti-H3K27me3 $((85) ; 0,5 \mathrm{mg} / \mathrm{ml})$ primary antibodies, all diluted in $150 \mu \mathrm{l}$ of $1 \times$ PBS/EDTA/1\% BSA (1×PBS/EDTA supplemented with $1 \% \mathrm{w} / \mathrm{v}$ bovine serum albumin), at $4^{\circ} \mathrm{C}$, overnight. All the above antibodies were applied to the wells at a titer of 1:800. After labeling with the primary antibodies, the nuclei were washed with $500 \mu 1$ ice cold $1 \times$ PBS/EDTA 3 times for 10 minutes. Labeling with the secondary antibodies was performed in $150 \mu 11 \times$ PBS/EDTA for two hours on ice, using Alexa fluor 488 (A488) or Alexa fluor 647 (A647) conjugated goat anti-mouse IgG or goat anti-rabbit IgG antibodies (Thermo Fisher Scientific, Waltham, Massachusetts, USA; $2 \mathrm{mg} / \mathrm{ml}$ ). In the case of sheep polyclonal anti-H2A.Z (acetyl K4+K7+K11, ab18262) Alexa fluor 633 (A633) conjugated goat anti-sheep IgG (Thermo Fisher Scientific, Waltham, Massachusetts, USA; $2 \mathrm{mg} / \mathrm{ml}$ ) secondary antibody was used. The secondary antibodies were also used at a titer of 1:800, diluted in $1 \times$ PBS/EDTA from $2 \mathrm{mg} / \mathrm{ml}$ stock solutions. After labeling with the secondary antibodies the agarose embedded nuclei were washed with $500 \mu 1$ ice cold $1 \times$ PBS/EDTA 3 times, for 10 minutes. Then the samples were fixed in $1 \%$ formaldehyde (dissolved in $1 \times \mathrm{PBS} / \mathrm{EDTA}$ ) at $4^{\circ} \mathrm{C}$, overnight. After fixation the wells containing the embedded nuclei were washed with $500 \mu$ ice cold $1 \times$ PBS/EDTA 3 times for 3 minutes and were stained with $200 \mu 1$ 12,5 $\mu \mathrm{g} / \mathrm{ml}$ propidium-iodide (PI, dissolved in $1 \times$ PBS/EDTA) for 30 minutes, on ice. The stained nuclei were washed 3 times with $500 \mu \mathrm{l}$ ice cold $1 \times$ PBS/EDTA for 3 minutes. Fluorescence intensity distributions were recorded using an iCys laser scanning cytometer (LSC), as described below.

\section{MNase, Nickase and DNase I treatment}

Live cells were embedded into agarose as described above and treated with $500 \mu \mathrm{l}$ ice cold lysis buffer $(0.4 \%$ (v/v) Triton X-100, $300 \mathrm{mM} \mathrm{NaCl}, 1 \mathrm{mM}$ EDTA, $10 \mathrm{mM}$ Tris-HCl, $\mathrm{pH}$ 8.0) for 10 minutes, followed by treatment with $500 \mu$ ice cold $1 \%(\mathrm{v} / \mathrm{v})$ Triton $\mathrm{X}-100$ dissolved in $1 \times$ PBS/EDTA, for 10 minutes, then washed 3 times with $500 \mu 1$ ice cold $1 \times$ PBS/EDTA. MNase, the frequent cutter Nt.CviPII nickase (recognition site: CCD; New England Biolabs Inc., Ipswich, Massachusetts, USA) and DNase I were applied after the washing steps following permeabilization. Before digestion, the samples were equilibrated with MNase buffer (1 $\mathrm{mM}$ $\mathrm{CaCl}_{2}, 50 \mathrm{mM}$ Tris-HCL pH 7.5), nickase buffer (10 mM Tris- $\mathrm{HCl} \mathrm{pH} 8.0,50 \mathrm{mM} \mathrm{NaCl}, 10 \mathrm{mM}$ $\mathrm{MgCl}_{2}, 1 \mathrm{mg} / \mathrm{ml} \mathrm{BSA}$ ) or with DNase I buffer (10 mM Tris-HCl pH 8.0, 0.1 mM CaCl $2,2.5 \mathrm{mM}$ $\mathrm{MgCl}_{2}$ ) by washing 3 times with $500 \mu \mathrm{l}$ of the buffer solutions. Nickase treatment was performed in $300 \mu \mathrm{l}$ nickase buffer for $30 \mathrm{~min}$ at $37^{\circ} \mathrm{C}$, using the enzyme at a final concentration of $0.5 \mathrm{U} / \mathrm{ml}$. MNase and DNase I digestion were performed in $300 \mu \mathrm{l}$ MNase, or DNase I buffer for $10 \mathrm{~min}$ at $37{ }^{\circ} \mathrm{C}$, at a final concentrations indicated on the figures. After enzymatic treatments, the samples were washed with $500 \mu \mathrm{l}$ ice cold $1 \times$ PBS/EDTA 3 times for 3 minutes.

\section{Immuno- cross-linking experiments}


Agarose embedded cells were permeabilized in $500 \mu \mathrm{l}$ ice cold lysis buffer $(0.4 \%(\mathrm{v} / \mathrm{v})$ Triton X$100,300 \mathrm{mM} \mathrm{NaCl}, 1 \mathrm{mM}$ EDTA, $10 \mathrm{mM}$ Tris-HCl, pH 8.0) for 10 minutes, followed by treatment with $500 \mu \mathrm{l}$ ice cold $1 \%(\mathrm{v} / \mathrm{v})$ Triton X-100 dissolved in $1 \times$ PBS/EDTA, for 10 minutes, then washed 3 times with $500 \mu$ ice cold $1 \times$ PBS/EDTA. Permeabilized cells were incubated with 500 $\mu 15 \%(\mathrm{~m} / \mathrm{v})$ Blotto Non-Fat Dry Milk dissolved in $1 \times$ PBS/EDTA, for 30 minutes, on ice, to decrease nonspecific binding. The blocking solution was washed out with $500 \mu$ ice cold $1 \times$ PBS/EDTA 3 times and indirect immunofluorescence labeling was performed using rabbit polyclonal anti-H2A.Z (ab97966, Abcam, Cambridge, UK; $1 \mathrm{mg} / \mathrm{ml}$ ) at a titer of 1:800, diluted in $150 \mu \mathrm{l}$ of $1 \times \mathrm{PBS} / \mathrm{EDTA} / 1 \% \mathrm{BSA}$, at $4^{\circ} \mathrm{C}$, overnight. After labeling with the anti-H2A.Z antibody, the nuclei were washed with $500 \mu \mathrm{l}$ ice cold $1 \times \mathrm{PBS} /$ EDTA 3 times. Anti-H2A.Z labeled histones were cross-linked with the secondary antibody in $150 \mu 11 \times$ PBS/EDTA for 2 hours on ice, using A647-conjugated goat anti-rabbit IgG. The secondary antibody was used at a titer of 1:800, diluted in $1 \times \mathrm{PBS} / \mathrm{EDTA}$ from its $2 \mathrm{mg} / \mathrm{ml}$ stock solution. After labeling with the secondary antibody, nuclei were washed with $500 \mu$ ice cold $1 \times$ PBS/EDTA 3 times and the samples were treated with $0.1 \mathrm{U} / \mathrm{ml}$ MNase as described above. Then the samples were immunofluorescently labeled with antibodies specific for heterochromatic histone modifications (mouse monoclonal anti-H3K9me3, or mouse monoclonal anti-H3K27me3), as described above. After labeling samples were fixed with formaldehyde, stained with propidium iodide, as described above. The fluorescence intensity distributions were recorded by LSC.

\section{Etoposide treatment}

Agarose embedded live cells were treated with etoposide (TEVA, Debrecen, Hungary) used at a final concentration of $25 \mu \mathrm{M}$. The drug was diluted in $300 \mu \mathrm{l}$ complete DMEM medium and the cells were incubated together with the drug at $37^{\circ} \mathrm{C}$ in $5 \% \mathrm{CO} 2$ atmosphere.

\section{Automated microscopy}

Automated microscopic imaging was performed using an iCys instrument (iCys® Research Imaging Cytometer; CompuCyte, Westwood, Massachusetts, USA). Green fluorescent protein (GFP), SYBR Gold, A488 and PI were excited using a $488 \mathrm{~nm}$ Argon ion laser, A647 with a 633 $\mathrm{nm}$ HeNe laser. Fluorescence signals were collected via an UPlan FI 20× (NA 0.5) objective, scanning each field with a step size of $1.5 \mu \mathrm{m}$. GFP and A488 were detected through 510/21 nm and 530/30 nm filters, respectively, while A647 and PI were detected through a 650/LP nm filter.

Data evaluation and hardware control were performed with the iCys 7.0 software for Windows XP. Gating of G1 phase cells was according to the fluorescence intensity distribution of the DNA labeled with PI or SYBR Gold.

\section{Confocal Laser Scanning Microscopy (CLSM)}

Confocal images were taken using an FLUOVIEW FV 1000 confocal microscope (Olympus, Center Valley, Pennsylvania, USA) based on an inverted IX-81 stand with an UPLS APO 60x 
(NA 1.35) oil immersion objective. GFP or A488 were excited by a $488 \mathrm{~nm}$ Argon ion laser. A647 and PI were excited by a $633 \mathrm{~nm}$ and $543 \mathrm{~nm}$ HeNe laser, respectively.

\section{Colocalization and texture analyzis}

For image and texture analyzes, the Just Another Colocalization Plugin of the Image J software (http://imagej.nih.gov/ij/) and the MeasureGranularity module of the CellProfiler 2.2.0 software, respectively, were used.

\section{Sample preparation for mass spectrometric (MS) measurements}

$80 \times 10^{6} \mathrm{H} 3$-GFP expressing HeLa cells in $2.5 \mathrm{ml}$ cell culture medium were mixed with an equal amount of $1 \%$ LMP diluted in $1 \times$ PBS. Agarose blocks were prepared where each blocks contained $90 \mu \mathrm{l}$ of agarose/cell suspension. The blocks were washed 3 times in $15 \mathrm{ml} 1 \times \mathrm{PBS}$ for $10 \mathrm{~min}$. Permeabilization of cells was performed in ice cold $15 \mathrm{ml} 1 \%$ Triton-X 100 diluted in 1×PBS/EDTA, twice for $30 \mathrm{~min}$. Blocks were washed 5 times in $15 \mathrm{ml} 1 \times$ PBS/EDTA. Blocks were treated with $15 \mathrm{ml} \mathrm{EBr}$ diluted in $1 \times \mathrm{PBS} / \mathrm{EDTA} / 600 \mathrm{mM} \mathrm{NaCl}$ at a concentration of 100 $\mu \mathrm{g} / \mathrm{ml}$, or with $15 \mathrm{ml} 1.2 \mathrm{M} \mathrm{NaCl}$ solution diluted in $1 \times \mathrm{PBS} / \mathrm{EDTA}$, for $60 \mathrm{~min}$. Blocks were washed 3 times in $15 \mathrm{ml} 1 \times \mathrm{PBS} / \mathrm{EDTA}$ for $20 \mathrm{~min}$. and the proteins that remained in the nuclei were eluted with $15 \mathrm{ml}$ of $2 \mathrm{M} \mathrm{NaCl}$ diluted in $1 \times \mathrm{PBS} /$ EDTA for $60 \mathrm{~min}$. All the washing steps were performed using ice cold solutions. Eluted proteins were concentrated using a 10K Amicon tube (Merck-Millipore, Darmstadt, Germany) and the buffer was changed to $1 \times \mathrm{PBS}$. Proteins were eluted from the filter with $250 \mu \mathrm{l} 1 \times \mathrm{PBS}$ and stored at $-20^{\circ} \mathrm{C}$ for MS analyzes.

\section{SDS-PAGE and LC-MS/MS analyzes}

The protein concentration of each sample was measured using the Bradford method (43). $20 \mu \mathrm{g}$ protein was analyzed on a $10 \%$ SDS polyacrylamide gel. Electrophoresis was carried out in a BioRad mini tetra cell (Bio-Rad) at 100 A constant current for one hour. The proteins were stained in the gel using Coomassie PageBlue (Fermentas) and scanned with a Pharos FX Plus laser scanner (Bio-Rad). The bands of SDS-PAGE were excised, followed by in-gel digestion with trypsin. Reduction was performed using $20 \mathrm{mM}$ dithiothreitol for one hour at $56^{\circ} \mathrm{C}$, followed by alkylation with $55 \mathrm{mM}$ iodoacetamide for $45 \mathrm{~min}$. Overnight trypsin digestion was carried out using stabilized MS grade TPCK-treated bovine trypsin (ABSciex) at $37{ }^{\circ} \mathrm{C}$; thereafter, the digested peptides were extracted and lyophilized. The peptides were re-dissolved in $10 \mu 11 \%$ formic acid before MS analyzes. The acquired LC-MS/MS data were used for protein identification with the help of Protein-Pilot 4.0 (ABSciex) search engine and the SwissProt database, using the biological modification table included in the ProteinPilot 4.0 software. A minimum of two peptide sequences with $95 \%$ confidence were used for protein identification. SDS-PAGE and MS analyzes were done at the Proteomics Core Facility of the Department of Biochemistry and Molecular Biology, University of Debrecen using an Orbitrap Fusion mass spectrometer (Thermo Fisher Scientific, Waltham, Massachusetts, USA). 


\section{ACKNOWLEDGEMENTS}

The authors thank Sandra B. Hake (Giessen, Germany) for the PWWP2A-GFP plasmid and for the gift of anti-H2A.Z antibody ab4174.

Zheng Zhou (Beijing, China) for explanations regarding their cryo-EM data on H2A.Z structure, Adel Vezendine Nagy for technical help. Mass spectrometry analysis was carried out at the BMBI Proteomics Core Facility, Department of Biochemistry and Molecular Biology, University of Debrecen.

\section{FUNDING STATEMENT}

GS received funding from GINOP-2.3.2-15-2016-00044, GINOP-2.3.3-15-2016-00020, Hungarian National Science and Research Foundation OTKA K128770 (https://nkfih.gov.hu/funding/otka), COST EuroCellNet CA15214 and CA18127 (https://www.eurocellnet.eu). The Orbitrap Fusion mass spectrometer was provided by GINOP2.3.3-15-2016-00020 for the Proteomics Core Facility. RB was supported by Stipendium Hungaricum awarded by the Tempus Public foundation (https://tka.hu/english). The funders had no role in study design, data collection and analysis, decision to publish, or preparation of the manuscript. JA research on this topic was supported by a Canadian Institutes of Health (CIHR) grant.

\section{CONTRIBUTIONS}

IL: LSC experiments, confocal microscopic studies, texture analyses, cytotoxicity measurements; MH and MK: microarray data; RB and ÁCs: bacterial production of plasmids; PN: analyses of microarray data provided by $\mathrm{MH}$; GSz: conceptualization of the experiments, writing of manuscript together with IL; MH: conceptualization of the experiments; GM supervised peptide synthesis condcuted by KNE; JA: advice and editing of the manuscript.

\section{REFERENCES}

1. Hatch, C.L. and Bonner, W.M. (1988) Sequence of cDNAs for mammalian H2A.Z, an evolutionarily diverged but highly conserved basal histone $\mathrm{H} 2 \mathrm{~A}$ isoprotein species. Nucleic acids research, 16, 1113-1124.

2. Colino-Sanguino, Y., Clark, S.J. and Valdes-Mora, F. (2016) H2A.Z acetylation and transcription: ready, steady, go! Epigenomics, 8, 583-586.

3. Giaimo, B.D., Ferrante, F., Herchenrother, A., Hake, S.B. and Borggrefe, T. (2019) The histone variant H2A.Z in gene regulation. Epigenetics \& chromatin, 12, 37.

4. Sura, W., Kabza, M., Karlowski, W.M., Bieluszewski, T., Kus-Slowinska, M., Paweloszek, L., Sadowski, J. and Ziolkowski, P.A. (2017) Dual Role of the Histone Variant H2A.Z in Transcriptional Regulation of Stress-Response Genes. The Plant cell, 29, 791807. 
5. Bagchi, D.N., Battenhouse, A.M., Park, D. and Iyer, V.R. (2020) The histone variant H2A.Z in yeast is almost exclusively incorporated into the +1 nucleosome in the direction of transcription. Nucleic acids research, 48, 157-170.

6. Cheema, M.S., Good, K.V., Kim, B., Soufari, H., O'Sullivan, C., Freeman, M.E., Stefanelli, G., Casas, C.R., Zengeler, K.E., Kennedy, A.J. et al. (2020) Deciphering the Enigma of the Histone H2A.Z-1/H2A.Z-2 Isoforms: Novel Insights and Remaining Questions. Cells, 9.

7. Long, H., Zhang, L., Lv, M., Wen, Z., Zhang, W., Chen, X., Zhang, P., Li, T., Chang, L., Jin, C. et al. (2020) H2A.Z facilitates licensing and activation of early replication origins. Nature, 577, 576-581.

8. Ryan, D.P. and Tremethick, D.J. (2018) The interplay between H2A.Z and H3K9 methylation in regulating HP1alpha binding to linker histone-containing chromatin. Nucleic acids research, 46, 9353-9366.

9. Becker, J.S., Nicetto, D. and Zaret, K.S. (2016) H3K9me3-Dependent Heterochromatin: Barrier to Cell Fate Changes. Trends in genetics : TIG, 32, 29-41.

10. Piquet, S., Le Parc, F., Bai, S.K., Chevallier, O., Adam, S. and Polo, S.E. (2018) The Histone Chaperone FACT Coordinates H2A.X-Dependent Signaling and Repair of DNA Damage. Molecular cell, 72, 888-901 e887.

11. Gursoy-Yuzugullu, O., Ayrapetov, M.K. and Price, B.D. (2015) Histone chaperone Anp32e removes H2A.Z from DNA double-strand breaks and promotes nucleosome reorganization and DNA repair. Proceedings of the National Academy of Sciences of the United States of America, 112, 7507-7512.

12. Hu, G., Cui, K., Northrup, D., Liu, C., Wang, C., Tang, Q., Ge, K., Levens, D., CraneRobinson, C. and Zhao, K. (2013) H2A.Z facilitates access of active and repressive complexes to chromatin in embryonic stem cell self-renewal and differentiation. Cell stem cell, 12, 180-192.

13. Rispal, J., Baron, L., Beaulieu, J.F., Chevillard-Briet, M., Trouche, D. and Escaffit, F. (2019) The H2A.Z histone variant integrates Wnt signaling in intestinal epithelial homeostasis. Nature communications, 10, 1827.

14. Domaschenz, R., Kurscheid, S., Nekrasov, M., Han, S. and Tremethick, D.J. (2017) The Histone Variant H2A.Z Is a Master Regulator of the Epithelial-Mesenchymal Transition. Cell reports, 21, 943-952.

15. Yang, B., Tong, R., Liu, H., Wu, J., Chen, D., Xue, Z., Ding, C., Zhou, L., Xie, H., Wu, J. et al. (2018) H2A.Z regulates tumorigenesis, metastasis and sensitivity to cisplatin in intrahepatic cholangiocarcinoma. International journal of oncology, 52, 1235-1245.

16. Giaimo, B.D., Ferrante, F., Vallejo, D.M., Hein, K., Gutierrez-Perez, I., Nist, A., Stiewe, T., Mittler, G., Herold, S., Zimmermann, T. et al. (2018) Histone variant H2A.Z deposition and acetylation directs the canonical Notch signaling response. Nucleic acids research, $\mathbf{4 6}$, 8197-8215.

17. Wang, J., Qiao, M., He, Q., Shi, R., Loh, S.J., Stanton, L.W. and Wu, M. (2015) Pluripotency Activity of Nanog Requires Biochemical Stabilization by Variant Histone Protein H2A.Z. Stem cells, 33, 2126-2134.

18. Taylor, R., Long, J., Yoon, J.W., Childs, R., Sylvestersen, K.B., Nielsen, M.L., Leong, K.F., Iannaccone, S., Walterhouse, D.O., Robbins, D.J. et al. (2019) Regulation of GLI1 by cis DNA elements and epigenetic marks. DNA repair, 79, 10-21.

19. Gretzinger, T.L., Tyagi, M., Fontaine, C.J., Cheema, M.S., Gonzalez-Perez, M., Freeman, M.E., Christie, B.R. and Ausio, J. (2019) Fetal alcohol spectrum disorder (FASD) affects 
the hippocampal levels of histone variant H2A.Z-2. Biochemistry and cell biology = Biochimie et biologie cellulaire, 97, 431-436.

20. Dunn, C.J., Sarkar, P., Bailey, E.R., Farris, S., Zhao, M., Ward, J.M., Dudek, S.M. and Saha, R.N. (2017) Histone Hypervariants H2A.Z.1 and H2A.Z.2 Play Independent and Context-Specific Roles in Neuronal Activity-Induced Transcription of Arc/Arg3.1 and Other Immediate Early Genes. eNeuro, 4.

21. Ramzan, F., Baumbach, J., Monks, A.D. and Zovkic, I.B. (2020) Histone H2A.Z is required for androgen receptor-mediated effects on fear memory. Neurobiology of learning and memory, 175, 107311.

22. Shen, T., Ji, F., Wang, Y., Lei, X., Zhang, D. and Jiao, J. (2018) Brain-specific deletion of histone variant H2A.z results in cortical neurogenesis defects and neurodevelopmental disorder. Nucleic acids research, 46, 2290-2307.

23. West, M.H. and Bonner, W.M. (1980) Histone 2A, a heteromorphous family of eight protein species. Biochemistry, 19, 3238-3245.

24. Fujii, T., Ueda, T., Nagata, S. and Fukunaga, R. (2010) Essential role of p400/mDomino chromatin-remodeling ATPase in bone marrow hematopoiesis and cell-cycle progression. The Journal of biological chemistry, 285, 30214-30223.

25. Liang, X., Shan, S., Pan, L., Zhao, J., Ranjan, A., Wang, F., Zhang, Z., Huang, Y., Feng, H., Wei, D. et al. (2016) Structural basis of H2A.Z recognition by SRCAP chromatinremodeling subunit YL1. Nature structural \& molecular biology, 23, 317-323.

26. Obri, A., Ouararhni, K., Papin, C., Diebold, M.L., Padmanabhan, K., Marek, M., Stoll, I., Roy, L., Reilly, P.T., Mak, T.W. et al. (2014) ANP32E is a histone chaperone that removes H2A.Z from chromatin. Nature, 505, 648-653.

27. Alatwi, H.E. and Downs, J.A. (2015) Removal of H2A.Z by INO80 promotes homologous recombination. EMBO reports, 16, 986-994.

28. Papamichos-Chronakis, M., Watanabe, S., Rando, O.J. and Peterson, C.L. (2011) Global regulation of H2A.Z localization by the INO80 chromatin-remodeling enzyme is essential for genome integrity. Cell, 144, 200-213.

29. Klein, D.C. and Hainer, S.J. (2020) Chromatin regulation and dynamics in stem cells. Current topics in developmental biology, 138, 1-71.

30. Hainer, S.J. and Fazzio, T.G. (2015) Regulation of Nucleosome Architecture and Factor Binding Revealed by Nuclease Footprinting of the ESC Genome. Cell reports, 13, 61-69.

31. Bonisch, C. and Hake, S.B. (2012) Histone H2A variants in nucleosomes and chromatin: more or less stable? Nucleic acids research, 40, 10719-10741.

32. Park, Y.J., Dyer, P.N., Tremethick, D.J. and Luger, K. (2004) A new fluorescence resonance energy transfer approach demonstrates that the histone variant $\mathrm{H} 2 \mathrm{AZ}$ stabilizes the histone octamer within the nucleosome. The Journal of biological chemistry, 279, 24274-24282.

33. Fan, J.Y., Gordon, F., Luger, K., Hansen, J.C. and Tremethick, D.J. (2002) The essential histone variant H2A.Z regulates the equilibrium between different chromatin conformational states. Nature structural biology, 9, 172-176.

34. Hoch, D.A., Stratton, J.J. and Gloss, L.M. (2007) Protein-protein Forster resonance energy transfer analysis of nucleosome core particles containing H2A and H2A.Z. Journal of molecular biology, 371, 971-988. 
35. Thambirajah, A.A., Dryhurst, D., Ishibashi, T., Li, A., Maffey, A.H. and Ausio, J. (2006) H2A.Z stabilizes chromatin in a way that is dependent on core histone acetylation. The Journal of biological chemistry, 281, 20036-20044.

36. Abbott, D.W., Ivanova, V.S., Wang, X., Bonner, W.M. and Ausio, J. (2001) Characterization of the stability and folding of H2A.Z chromatin particles: implications for transcriptional activation. The Journal of biological chemistry, 276, 41945-41949.

37. Henikoff, S. (2009) Labile H3.3+H2A.Z nucleosomes mark 'nucleosome-free regions'. Nature genetics, 41, 865-866.

38. Jin, C., Zang, C., Wei, G., Cui, K., Peng, W., Zhao, K. and Felsenfeld, G. (2009) H3.3/H2A.Z double variant-containing nucleosomes mark 'nucleosome-free regions' of active promoters and other regulatory regions. Nature genetics, 41, 941-945.

39. Rudnizky, S., Bavly, A., Malik, O., Pnueli, L., Melamed, P. and Kaplan, A. (2016) H2A.Z controls the stability and mobility of nucleosomes to regulate expression of the LH genes. Nat Commun, 7, 12958.

40. Murphy, K.E., Meng, F.W., Makowski, C.E. and Murphy, P.J. (2020) Genome-wide chromatin accessibility is restricted by ANP32E. Nature communications, 11, 5063.

41. Wen, Z., Zhang, L., Ruan, H. and Li, G. (2020) Histone variant H2A.Z regulates nucleosome unwrapping and CTCF binding in mouse ES cells. Nucleic acids research, $\mathbf{4 8 ,}$ 5939-5952.

42. Sarcinella, E., Zuzarte, P.C., Lau, P.N., Draker, R. and Cheung, P. (2007) Monoubiquitylation of H2A.Z distinguishes its association with euchromatin or facultative heterochromatin. Molecular and cellular biology, 27, 6457-6468.

43. Matsuda, R., Hori, T., Kitamura, H., Takeuchi, K., Fukagawa, T. and Harata, M. (2010) Identification and characterization of the two isoforms of the vertebrate H2A.Z histone variant. Nucleic acids research, 38, 4263-4273.

44. Dryhurst, D., Ishibashi, T., Rose, K.L., Eirin-Lopez, J.M., McDonald, D., Silva-Moreno, B., Veldhoen, N., Helbing, C.C., Hendzel, M.J., Shabanowitz, J. et al. (2009) Characterization of the histone H2A.Z-1 and H2A.Z-2 isoforms in vertebrates. BMC biology, 7, 86.

45. Faast, R., Thonglairoam, V., Schulz, T.C., Beall, J., Wells, J.R., Taylor, H., Matthaei, K., Rathjen, P.D., Tremethick, D.J. and Lyons, I. (2001) Histone variant H2A.Z is required for early mammalian development. Current biology : CB, 11, 1183-1187.

46. Greenberg, R.S., Long, H.K., Swigut, T. and Wysocka, J. (2019) Single Amino Acid Change Underlies Distinct Roles of H2A.Z Subtypes in Human Syndrome. Cell, 178, 1421-1436 e1424.

47. Tang, S., Huang, X., Wang, X., Zhou, X., Huang, H., Qin, L., Tao, H., Wang, Q. and Tao, Y. (2020) Vital and Distinct Roles of H2A.Z Isoforms in Hepatocellular Carcinoma. OncoTargets and therapy, 13, 4319-4337.

48. Suto, R.K., Clarkson, M.J., Tremethick, D.J. and Luger, K. (2000) Crystal structure of a nucleosome core particle containing the variant histone H2A.Z. Nature structural biology, 7, 1121-1124.

49. Bonisch, C., Schneider, K., Punzeler, S., Wiedemann, S.M., Bielmeier, C., Bocola, M., Eberl, H.C., Kuegel, W., Neumann, J., Kremmer, E. et al. (2012) H2A.Z.2.2 is an alternatively spliced histone $\mathrm{H} 2 \mathrm{~A} . \mathrm{Z}$ variant that causes severe nucleosome destabilization. Nucleic acids research, 40, 5951-5964. 
50. Imre, L., Simandi, Z., Horvath, A., Fenyofalvi, G., Nanasi, P., Niaki, E.F., Hegedus, E., Bacso, Z., Weyemi, U., Mauser, R. et al. (2017) Nucleosome stability measured in situ by automated quantitative imaging. Scientific reports, 7, 12734.

51. Kusakabe, M., Oku, H., Matsuda, R., Hori, T., Muto, A., Igarashi, K., Fukagawa, T. and Harata, M. (2016) Genetic complementation analysis showed distinct contributions of the $\mathrm{N}$-terminal tail of H2A.Z to epigenetic regulations. Genes to cells : devoted to molecular \& cellular mechanisms, 21, 122-135.

52. Punzeler, S., Link, S., Wagner, G., Keilhauer, E.C., Kronbeck, N., Spitzer, R.M., Leidescher, S., Markaki, Y., Mentele, E., Regnard, C. et al. (2017) Multivalent binding of PWWP2A to H2A.Z regulates mitosis and neural crest differentiation. The EMBO journal, 36, 2263-2279.

53. Zhou, M., Dai, L., Li, C., Shi, L., Huang, Y., Guo, Z., Wu, F., Zhu, P. and Zhou, Z. (2021) Structural basis of nucleosome dynamics modulation by histone variants H2A.B and H2A.Z.2.2. The EMBO journal, 40, e105907.

54. Bosire, R., Nanasi, P., Jr., Imre, L., Dienes, B., Szoor, A., Mazlo, A., Kovacs, A., Seidel, R., Vamosi, G. and Szabo, G. (2019) Intercalation of small molecules into DNA in chromatin is primarily controlled by superhelical constraint. PloS one, 14, e 0224936.

55. Wratting, D., Thistlethwaite, A., Harris, M., Zeef, L.A. and Millar, C.B. (2012) A conserved function for the H2A.Z C terminus. The Journal of biological chemistry, 287, 19148-19157.

56. Wang, A.Y., Aristizabal, M.J., Ryan, C., Krogan, N.J. and Kobor, M.S. (2011) Key functional regions in the histone variant H2A.Z C-terminal docking domain. Molecular and cellular biology, 31, 3871-3884.

57. Latrick, C.M., Marek, M., Ouararhni, K., Papin, C., Stoll, I., Ignatyeva, M., Obri, A., Ennifar, E., Dimitrov, S., Romier, C. et al. (2016) Molecular basis and specificity of H2A.Z-H2B recognition and deposition by the histone chaperone YL1. Nature structural \& molecular biology, 23, 309-316.

58. Kemble, D.J., McCullough, L.L., Whitby, F.G., Formosa, T. and Hill, C.P. (2015) FACT Disrupts Nucleosome Structure by Binding H2A-H2B with Conserved Peptide Motifs. Molecular cell, 60, 294-306.

59. Sevilla, A. and Binda, O. (2014) Post-translational modifications of the histone variant H2AZ. Stem cell research, 12, 289-295.

60. Foster, E.R. and Downs, J.A. (2009) Methylation of H3 K4 and K79 is not strictly dependent on H2B K123 ubiquitylation. The Journal of cell biology, 184, 631-638.

61. Kumar, A. and Kono, H. (2020) Heterochromatin protein 1 (HP1): interactions with itself and chromatin components. Biophysical reviews, 12, 387-400.

62. Watanabe, S., Mishima, Y., Shimizu, M., Suetake, I. and Takada, S. (2018) Interactions of HP1 Bound to H3K9me3 Dinucleosome by Molecular Simulations and Biochemical Assays. Biophysical journal, 114, 2336-2351.

63. Jacobs, S.A. and Khorasanizadeh, S. (2002) Structure of HP1 chromodomain bound to a lysine 9-methylated histone H3 tail. Science, 295, 2080-2083.

64. Mishima, Y., Watanabe, M., Kawakami, T., Jayasinghe, C.D., Otani, J., Kikugawa, Y., Shirakawa, M., Kimura, H., Nishimura, O., Aimoto, S. et al. (2013) Hinge and chromoshadow of HP1alpha participate in recognition of K9 methylated histone $\mathrm{H} 3$ in nucleosomes. Journal of molecular biology, 425, 54-70. 
65. Sanulli, S. and G, J.N. (2020) Liquid-like interactions in heterochromatin: Implications for mechanism and regulation. Current opinion in cell biology, 64, 90-96.

66. Sanulli, S., Trnka, M.J., Dharmarajan, V., Tibble, R.W., Pascal, B.D., Burlingame, A.L., Griffin, P.R., Gross, J.D. and Narlikar, G.J. (2019) HP1 reshapes nucleosome core to promote phase separation of heterochromatin. Nature, 575, 390-394.

67. Hao, F., Murphy, K.J., Kujirai, T., Kamo, N., Kato, J., Koyama, M., Okamato, A., Hayashi, G., Kurumizaka, H. and Hayes, J.J. (2020) Acetylation-modulated communication between the H3 N-terminal tail domain and the intrinsically disordered H1 C-terminal domain. Nucleic acids research, 48, 11510-11520.

68. Verni, F. and Cenci, G. (2015) The Drosophila histone variant H2A.V works in concert with HP1 to promote kinetochore-driven microtubule formation. Cell cycle, 14, 577-588.

69. Turner, A.L., Watson, M., Wilkins, O.G., Cato, L., Travers, A., Thomas, J.O. and Stott, K. (2018) Highly disordered histone H1-DNA model complexes and their condensates. Proceedings of the National Academy of Sciences of the United States of America, 115, 11964-11969.

70. Zhou, B.R., Feng, H., Kato, H., Dai, L., Yang, Y., Zhou, Y. and Bai, Y. (2013) Structural insights into the histone H1-nucleosome complex. Proceedings of the National Academy of Sciences of the United States of America, 110, 19390-19395.

71. Thakar, A., Gupta, P., Ishibashi, T., Finn, R., Silva-Moreno, B., Uchiyama, S., Fukui, K., Tomschik, M., Ausio, J. and Zlatanova, J. (2009) H2A.Z and H3.3 histone variants affect nucleosome structure: biochemical and biophysical studies. Biochemistry, 48, 1085210857.

72. White, A.E., Hieb, A.R. and Luger, K. (2016) A quantitative investigation of linker histone interactions with nucleosomes and chromatin. Scientific reports, 6, 19122.

73. Greaves, I.K., Rangasamy, D., Ridgway, P. and Tremethick, D.J. (2007) H2A.Z contributes to the unique 3D structure of the centromere. Proceedings of the National Academy of Sciences of the United States of America, 104, 525-530.

74. Creyghton, M.P., Markoulaki, S., Levine, S.S., Hanna, J., Lodato, M.A., Sha, K., Young, R.A., Jaenisch, R. and Boyer, L.A. (2008) H2AZ is enriched at polycomb complex target genes in ES cells and is necessary for lineage commitment. Cell, 135, 649-661.

75. Canzio, D., Liao, M., Naber, N., Pate, E., Larson, A., Wu, S., Marina, D.B., Garcia, J.F., Madhani, H.D., Cooke, R. et al. (2013) A conformational switch in HP1 releases autoinhibition to drive heterochromatin assembly. Nature, 496, 377-381.

76. Puc, J., Aggarwal, A.K. and Rosenfeld, M.G. (2017) Physiological functions of programmed DNA breaks in signal-induced transcription. Nature reviews. Molecular cell biology, 18, 471-476.

77. Wood, T.J., Thistlethwaite, A., Harris, M.R., Lovell, S.C. and Millar, C.B. (2013) Mutations in non-acid patch residues disrupt H2A.Z's association with chromatin through multiple mechanisms. PloS one, 8, e76394.

78. Belotti, E., Lacoste, N., Simonet, T., Papin, C., Padmanabhan, K., Scionti, I., Gangloff, Y.G., Ramos, L., Dalkara, D., Hamiche, A. et al. (2020) H2A.Z is dispensable for both basal and activated transcription in post-mitotic mouse muscles. Nucleic acids research, 48, 4601-4613.

79. Lee, S.K. and Wang, W. (2019) Roles of Topoisomerases in Heterochromatin, Aging, and Diseases. Genes, 10. 
80. Gursoy-Yuzugullu, O., House, N. and Price, B.D. (2016) Patching Broken DNA: Nucleosome Dynamics and the Repair of DNA Breaks. Journal of molecular biology, 428, 1846-1860.

81. Kachnic, L.A., Li, L., Fournier, L., Ferraiolo, N., Dahm-Daphi, J., Borgmann, K. and Willers, H. (2011) FANCD2 but not FANCA promotes cellular resistance to type II topoisomerase poisons. Cancer letters, 305, 86-93.

82. Srivatsan, A., Li, B.Z., Szakal, B., Branzei, D., Putnam, C.D. and Kolodner, R.D. (2018) The Swr1 chromatin-remodeling complex prevents genome instability induced by replication fork progression defects. Nature communications, 9, 3680.

83. Kimura, H. and Cook, P.R. (2001) Kinetics of Core Histones in Living Human Cells: Little Exchange of H3 and H4 and Some Rapid Exchange of H2B. The Journal of Cell Biology, 153, 1341-1353.

84. Kimura, H., Hayashi-Takanaka, Y., Goto, Y., Takizawa, N. and Nozaki, N. (2008) The organization of histone $\mathrm{H} 3$ modifications as revealed by a panel of specific monoclonal antibodies. Cell structure and function, 33, 61-73.

85. Hayashi-Takanaka, Y., Yamagata, K., Wakayama, T., Stasevich, T.J., Kainuma, T., Tsurimoto, T., Tachibana, M., Shinkai, Y., Kurumizaka, H., Nozaki, N. et al. (2011) Tracking epigenetic histone modifications in single cells using Fab-based live endogenous modification labeling. Nucleic acids research, 39, 6475-6488. 

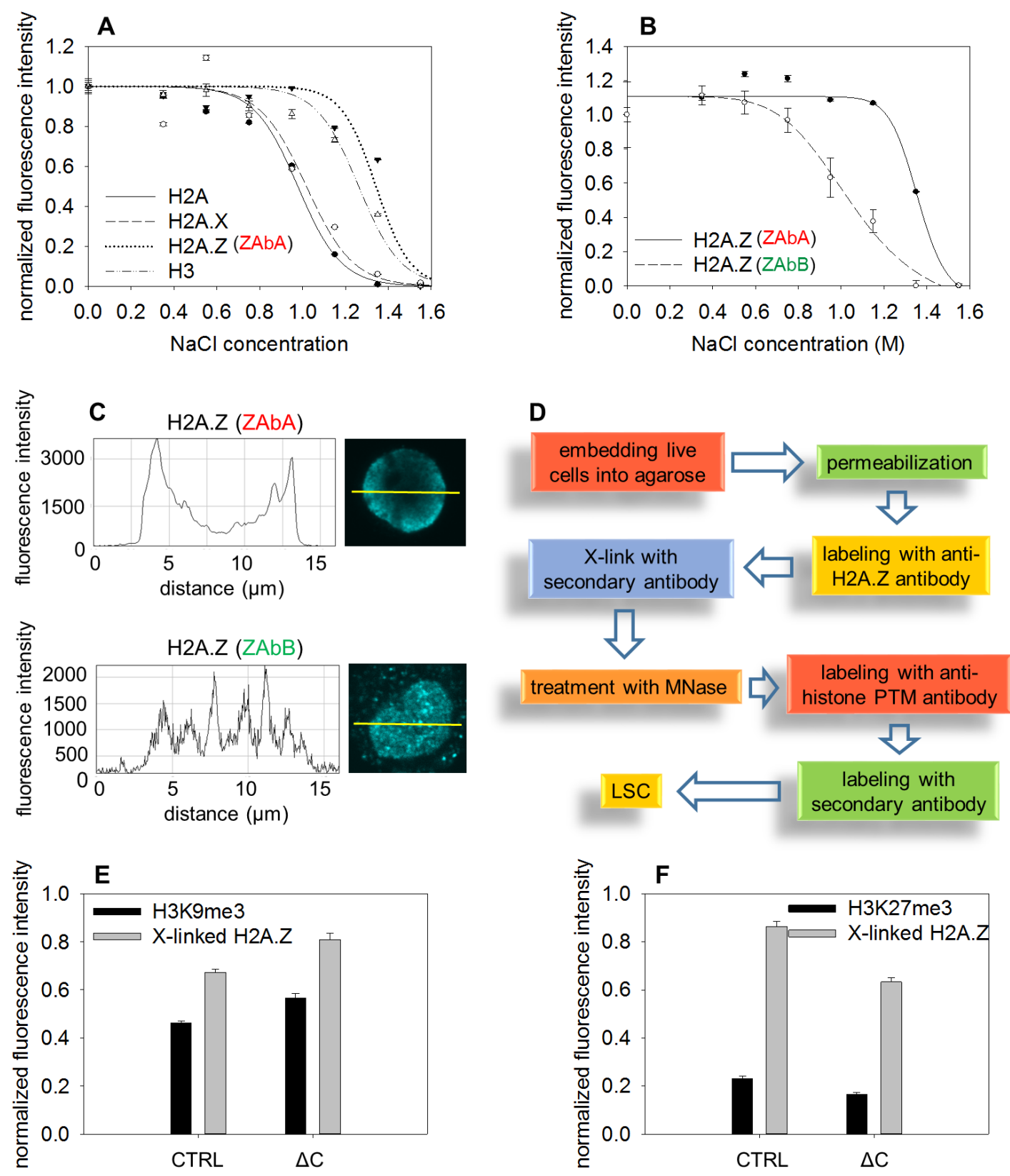

Figure 1. Intranuclear heterogeneity of H2A.Z-containing nucleosomes.

(A) Comparison of the salt elution profiles of H2A, H2A.X, H2A.Z detected by ZAbA (Abcam, ab 97966) antibody and of H3-GFP (used as an internal control) in HeLa cell nuclei. (B) Salt elution curves of H2A.Z detected by H2A.Z specific polyclonal antibodies from different manufacturers: ZAbA, ZAbB (Thermo Fisher Sci., PA5-17336). (C) CLSM images and line-scans showing nuclear 
localization of H2A.Z recognized by the ZAbA antibody (upper panel), or by ZAbB (lower panel). (D) Flow chart of the immuno-cross-linking (immune-X-linking) experiment in panels E, F. (E and F) immune-X-linking of H2A.Z immobilizes the H3K9me3 containing nucleosomes (E), in contrast with the H3K27me3 containing nucleosomes $(\mathrm{F})$, measured in H2A.Z1 $\Delta \mathrm{C}(\Delta \mathrm{C})$ and H2A.Z1 (control; CTRL) expressing DKO DT40 cells. 


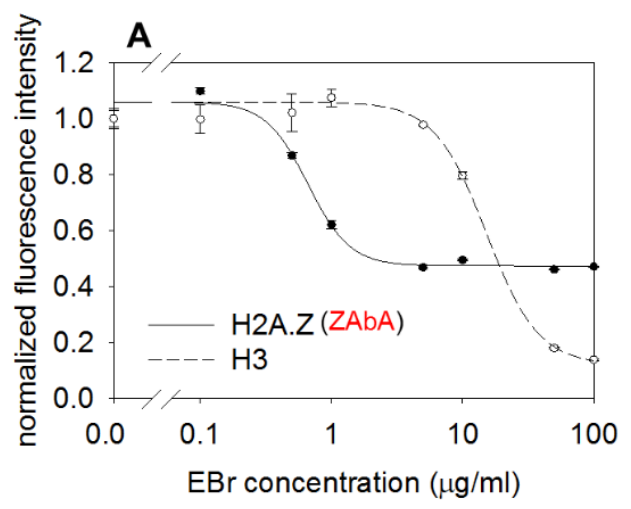

\section{C}

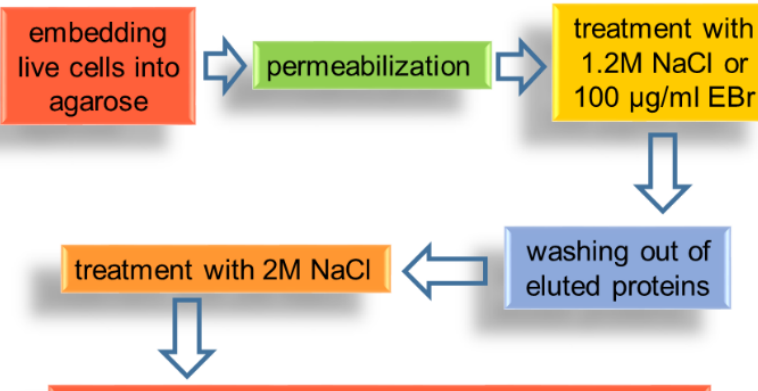

analyzis of eluted proteins by mass spectrometer
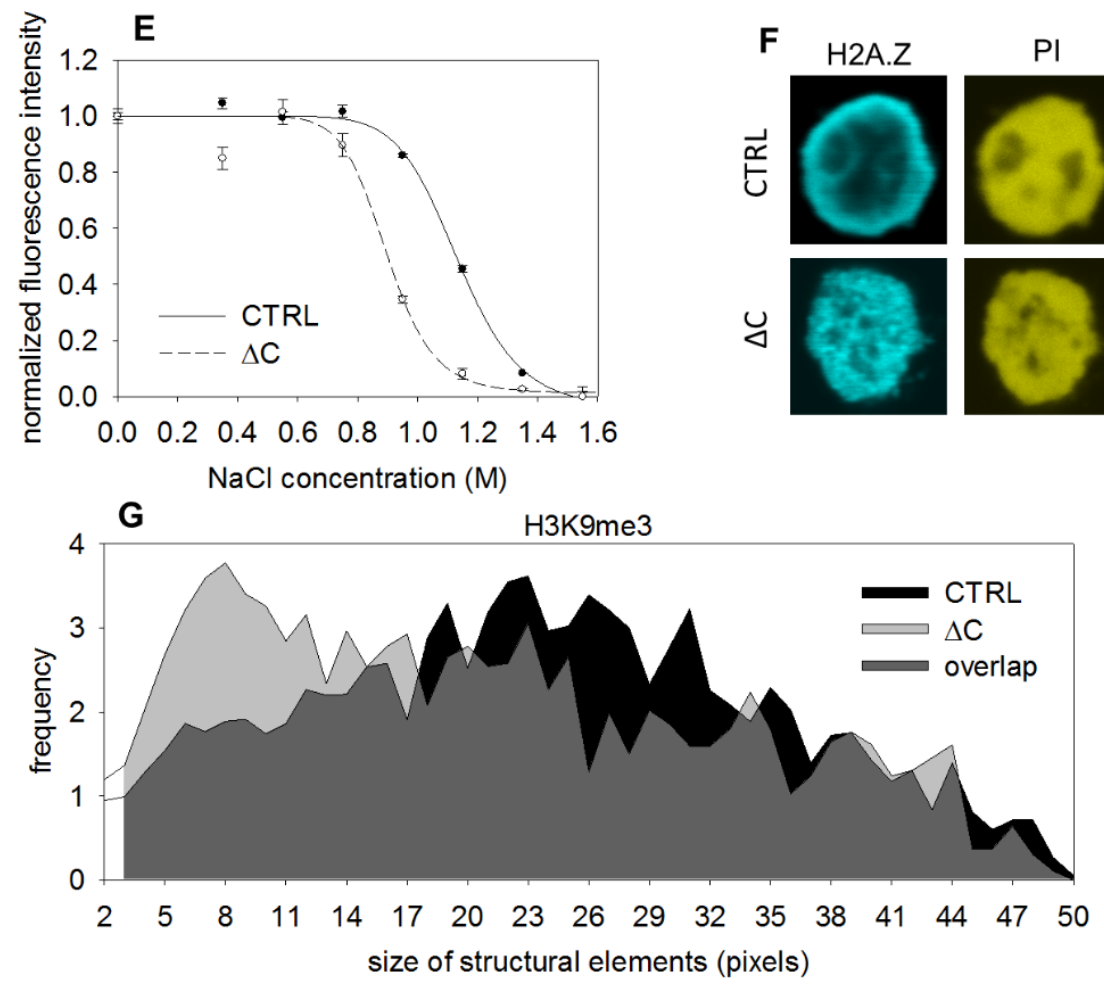

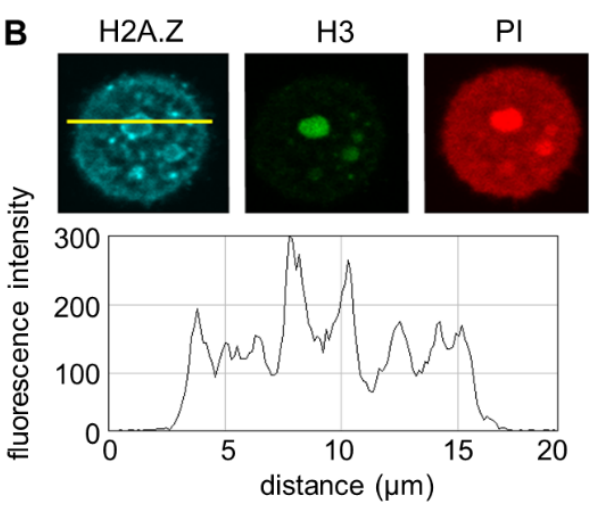

D $\quad \mathrm{NaCl}$ resistant

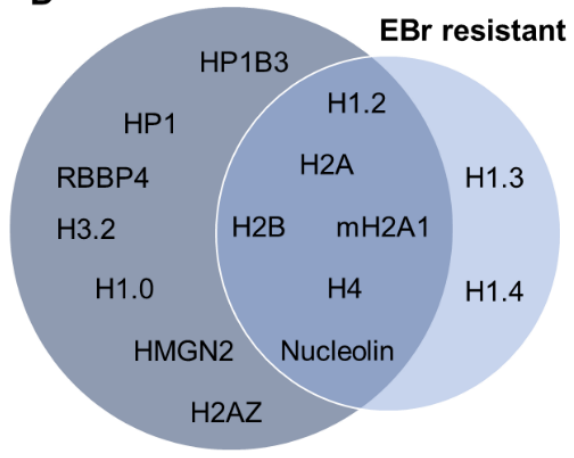

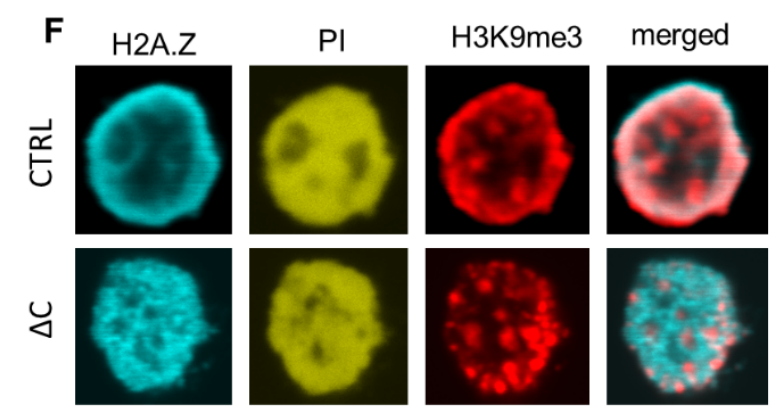

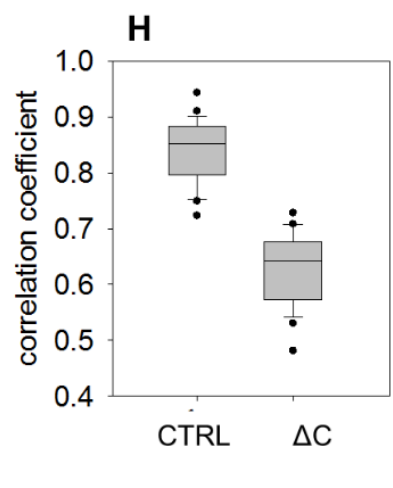


Figure 2. Mass spectrometric analyzes of salt or intercalator resistant chromatin fractions (A-D), and the effect of C-terminal truncation on the salt sensitivity and nuclear localization of H2A.Z and $\mathrm{H} 3 \mathrm{~K} 9 \mathrm{me} 3$ nucleosomes (E-H).

(A) EBr elution profile of H2A.Z detected by ZAbA compared to GFP-tagged H3 used as an internal control in HeLa nuclei. (B) CLSM image and line scan of H2A.Z after $100 \mu \mathrm{g} / \mathrm{ml}$ EBr treatment. EBr elution was performed in the presence of $0.75 \mathrm{M} \mathrm{NaCl}$ (50). (C) Flow chart of the elution experiment combined with mass spectrometry (MS). Proteins resistant to $1.2 \mathrm{M}$ salt or $100 \mu \mathrm{g} / \mathrm{ml}$ EBr treatment were detected by MS after high salt (2 M) elution. (D) Proteins identified by MS in the salt or intercalator resistant fraction known to be chromatin associated are listed in the Venn diagram. (E) Salt elution profiles of nucleosomes in H2A.Z1 $\Delta \mathrm{C}(\Delta \mathrm{C})$ and H2A.Z1 expressing DKO DT40 cells (CTRL), detected by ZAbA. (F) Representative CLSM images showing the nuclear localization of H2A.Z recognized by the ZAbA antibody, and H3K9me3 co-labeled with H2A.Z in DKO H2A.Z1 $\Delta \mathrm{C}$ and H2A.Z1 nuclei. (G) Texture analyzes (see Materials and Methods) of H2A.Z1 $\Delta \mathrm{C}(\Delta \mathrm{C})$ and H2A.Z1 (CTRL) expressing DKO DT40 nuclei showing the size distribution of structural elements containing $\mathrm{H} 3 \mathrm{~K} 9 \mathrm{me} 3$. $(\mathrm{H})$ Colocalization analyzes of H2A.Z and H3K9me3 in H2A.Z1 $\Delta \mathrm{C}(\Delta \mathrm{C})$ and H2A.Z1 (CTRL) nuclei. The Mandels colocalization coefficient reflecting the fraction of H2A.Z overlapping with $\mathrm{H} 3 \mathrm{~K} 9 \mathrm{me} 3$ is shown. Box-and-whisker plot was created from the data of 20-30 nuclei. 

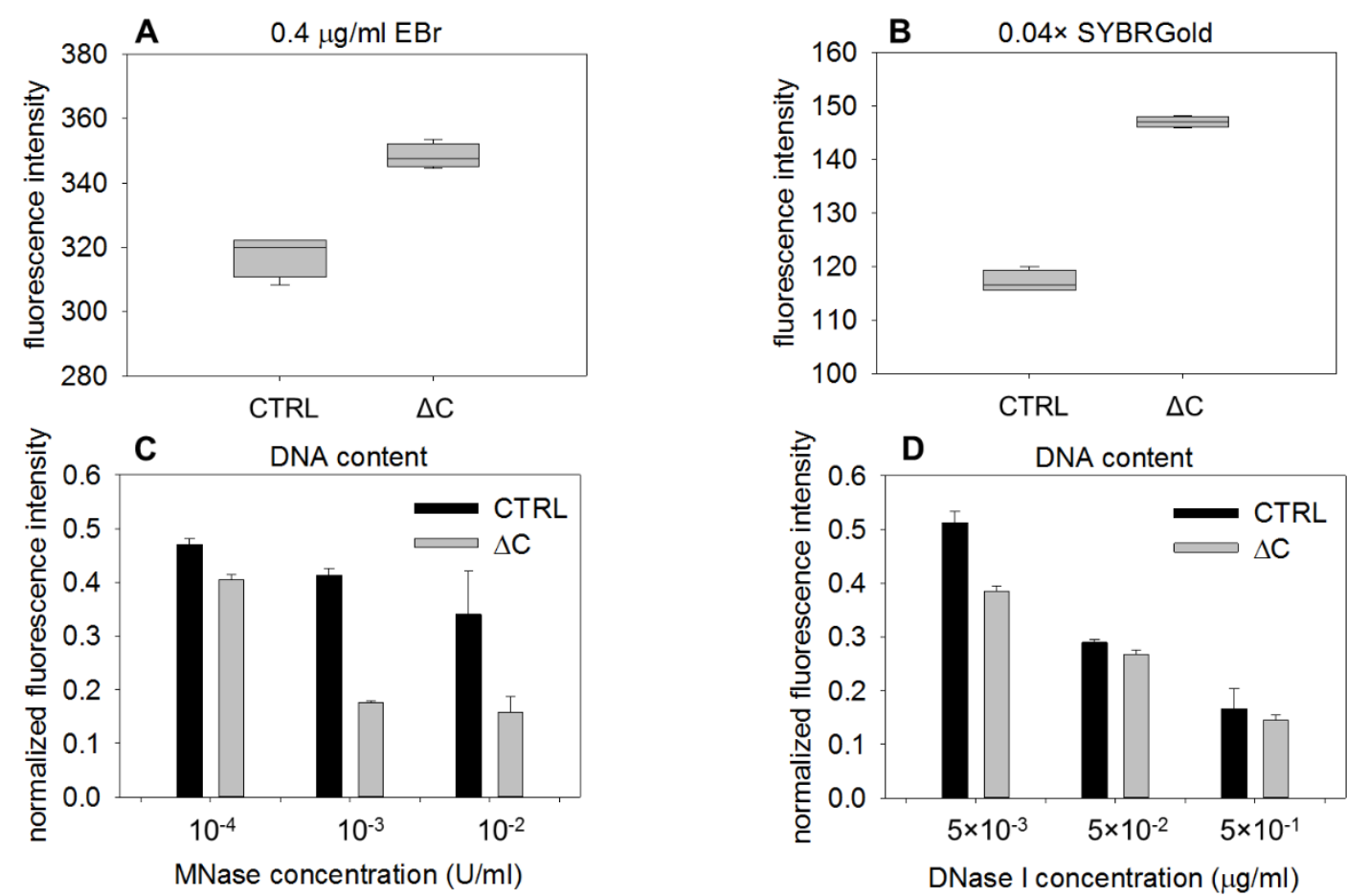
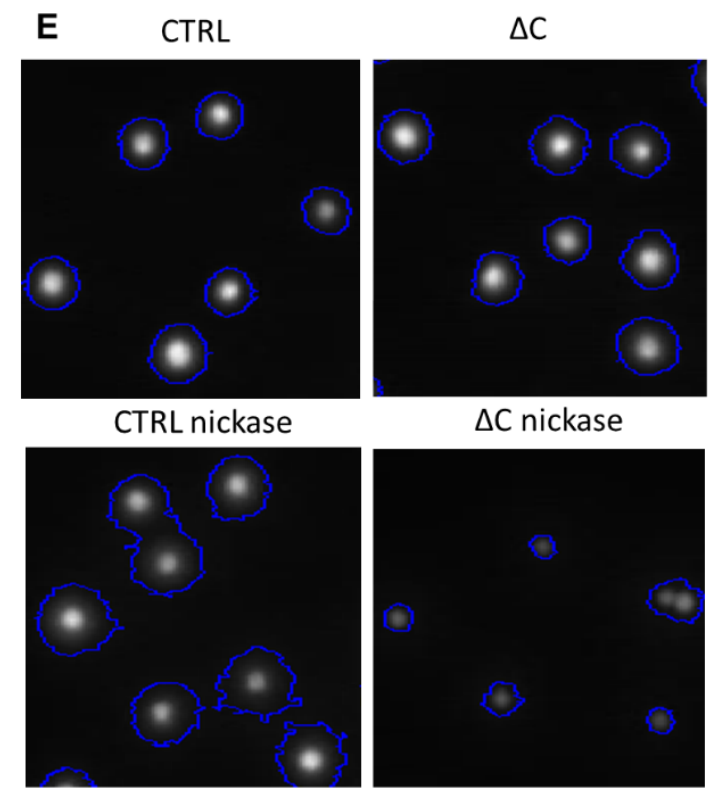

$\Delta C$ nickase

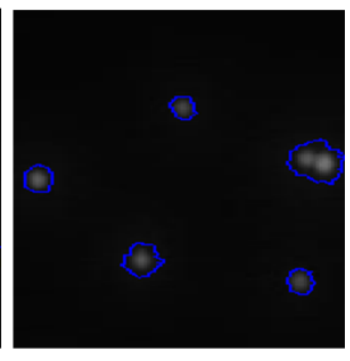

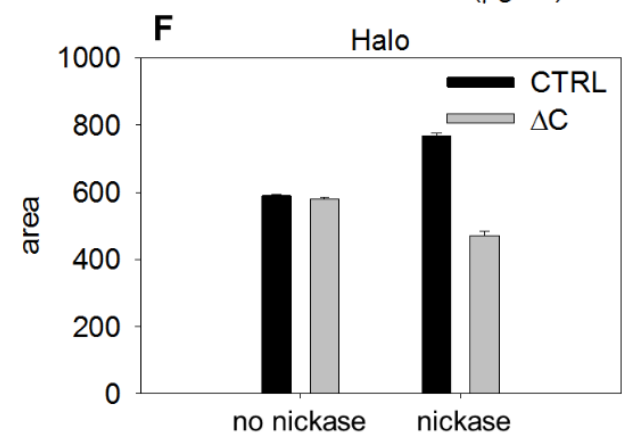

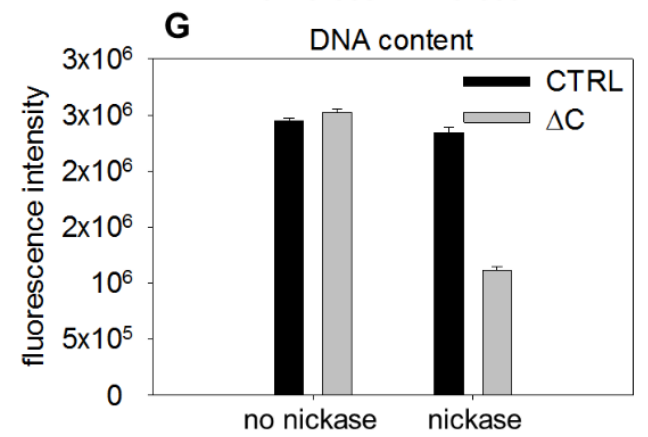

Figure 3. Global differences in chromatin accessibility features between DKO H2A.Z1 $\Delta \mathrm{C}$ and H2A.Z1 nuclei.

(A and B) Increased amount of intercalated $\mathrm{EBr}(\mathrm{A})$ and SYBRGold (B) was measured in H2A.Z $\triangle \mathrm{C}$ $(\triangle \mathrm{C})$ as compared to H2A.Z1 (CTRL) by flow-cytometric analyzes. Box-and-whisker plot was 
created from the mean fluorescence intensities of 4 parallel measurements. (C) and (D) Comparison of the sensitivity of chromatin to MNase (C) and DNase I (D). The DNA content of H2A.Z1 $\Delta \mathrm{C}$ $(\triangle \mathrm{C})$ and H2A.Z1 (CTRL) nuclei was measured by LSC after endonuclease treatment. (E-G) Comparison of the sensitivity of chromatin to a nickase. Halo size (E and F) and DNA content $(G)$ of H2A.Z1 $\Delta \mathrm{C}(\Delta \mathrm{C})$ and H2A.Z1 (CTRL) nuclei were measured by LSC after $0.5 \mathrm{U} / \mathrm{ml}$ nickase treatment.
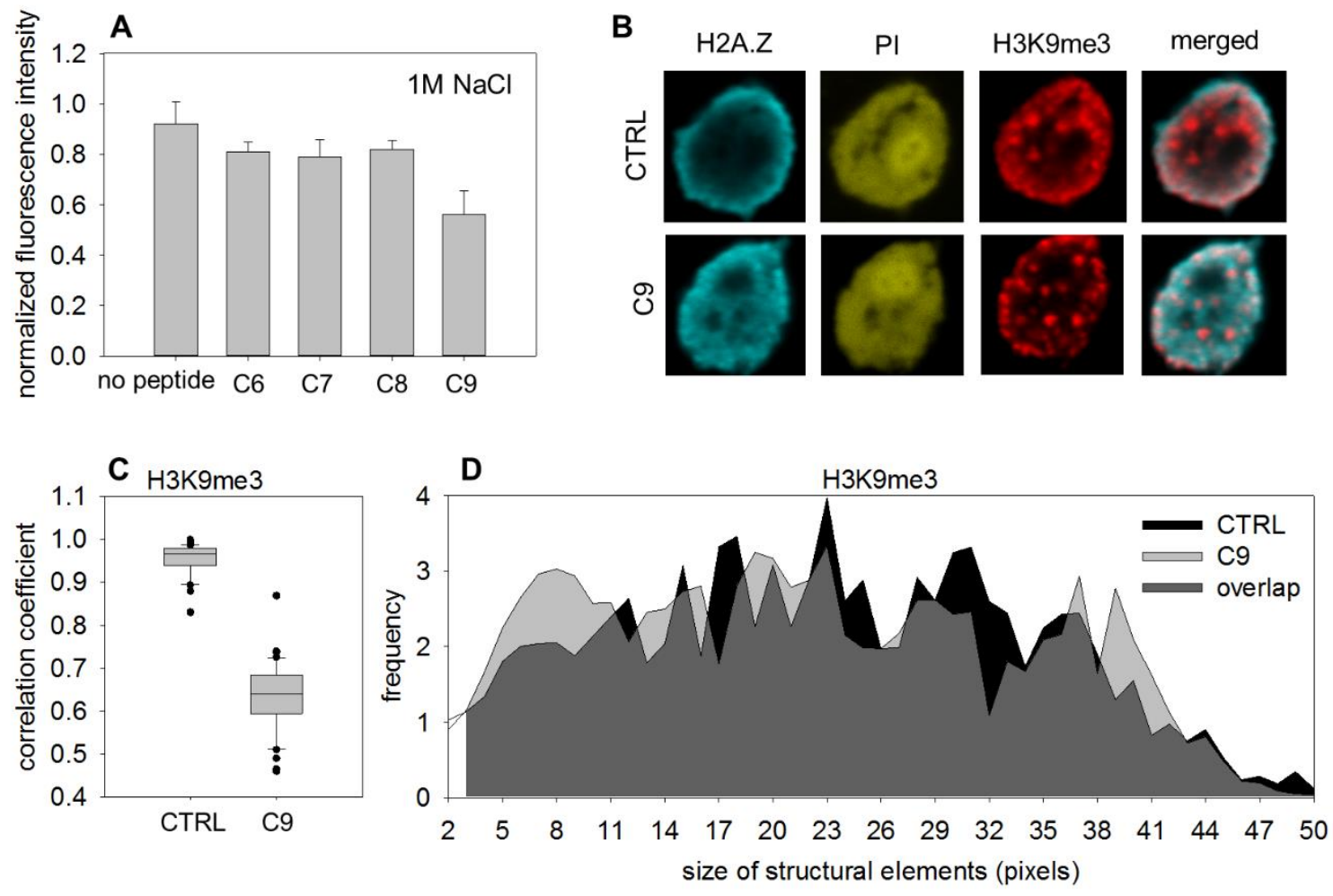

Figure 4. The effect of peptides containing the last 6-9 amino acids of the H2A.Z1 C-terminus on the stability and nuclear localization of H2A.Z1 and H3K9me3.

(A) Stability of H2A.Z nucleosomes in permeabilized HeLa nuclei exposed to $1 \mathrm{M} \mathrm{NaCl}$ in the presence of different length peptides (C6-C9). (B) Representative CLSM images showing nuclear localization of $\mathrm{H} 2 \mathrm{~A} . \mathrm{Z}$ recognized by the ZAbA antibody, or $\mathrm{H} 3 \mathrm{~K} 9 \mathrm{me} 3$ co-labeled with $\mathrm{H} 2 \mathrm{~A} . \mathrm{Z}$ in control (CTRL) or C9 treated (C9) DKO H2A.Z1 nuclei. (C) Colocalization analyzes of H2A.Z and H3K9me3 comparing DKO H2A.Z1 nuclei before (CTRL) or after the addition of the C9 peptide (C9). The change of the Mandels colocalization coefficient representing the fraction of H2A.Z 
overlapping with H3K9me3 is shown. Box-and-whisker plot was created from the data of 20-30 nuclei. (D) Texture analyzes of control (CTRL) and C9 treated (C9) DKO H2A.Z1 nuclei showing the size distribution of structural elements containing H3K9me3.
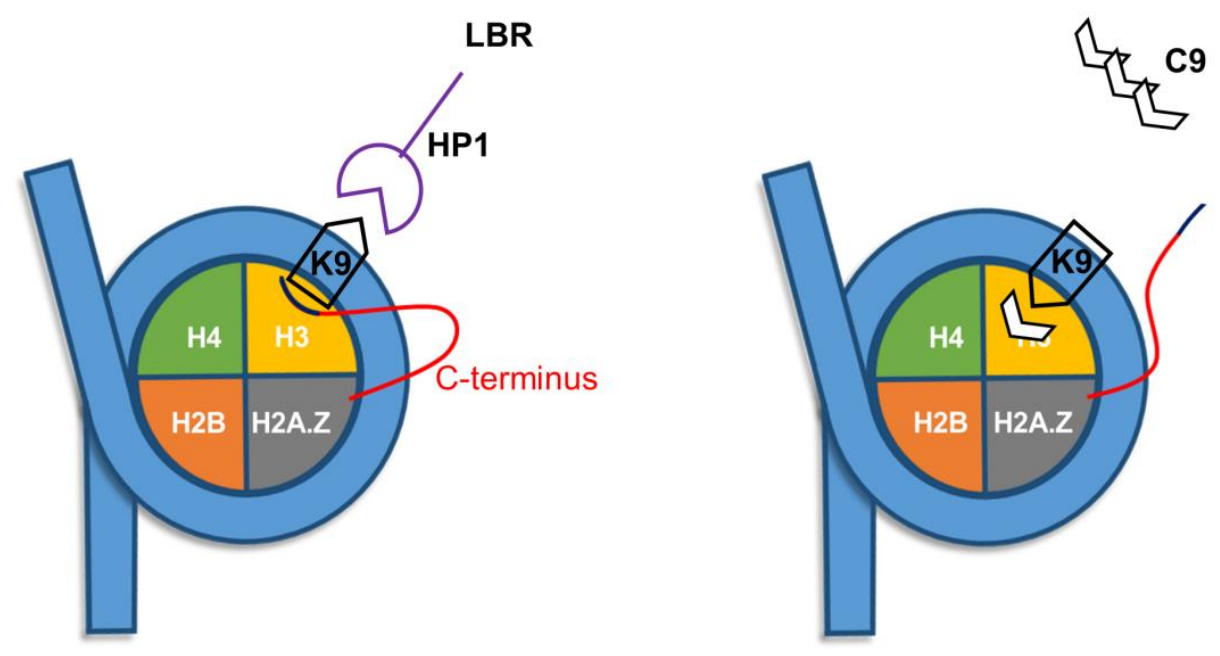

Figure 5. Modell for the role of H2A.Z C-terminus in the determination of nucleosome stability and intranuclear localization.

The fraction of nucleosomes with homotypic H2A.Z content and H3K9me3 PTM are represented in the scheme. The H2A.Z1 C-terminal tail is shown by a curved red line, with its last 9 amino acids painted black. The N-terminal H3 tail carrying K9me3 is drawn as an object ending in a triangle, shown engaged in binding to HP1. HP1 is thought to be tethered to the nuclear lamina via the lamin binding receptor (LBR). The C9 peptide molecules added to the nuclei in the experiment shown in Fig. 4. are shown as flat arrow-shaped objects. The model assumes that the H2A.Z tail binds to H3 in such a manner that allows H3K9me3 interact with HP1, stabilizing as well as anchoring the nucleosome to the periphery of the nucleus, and that both of these features are simultaneously cancelled when the C9 peptide occupies the binding site of the tail on $\mathrm{H} 3$. As a consequence, the stability features will become similar to those of the canonical nucleosomes and these nucleosomes will lose their lamina anchorage. Nucleosomes containing H3K9me3 alone will remain laminaassociated and are not represented in the scheme. 